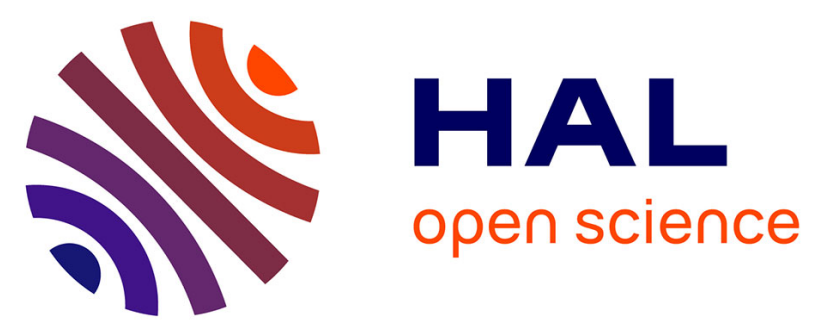

\title{
Free cholesterol transfer to high-density lipoprotein (HDL) upon triglyceride lipolysis underlies the U-shape relationship between HDL-cholesterol and cardiovascular disease
}

\author{
Ma Feng, Maryam Darabi, Emilie Tubeuf, Aurélie Canicio, Marie Lhomme, \\ Eric Frisdal, Sandrine Lanfranchi-Lebreton, Lucrèce Mathéron, Fabiana \\ Rached, Maharajah Ponnaiah, et al.
}

\section{- To cite this version:}

Ma Feng, Maryam Darabi, Emilie Tubeuf, Aurélie Canicio, Marie Lhomme, et al.. Free cholesterol transfer to high-density lipoprotein (HDL) upon triglyceride lipolysis underlies the U-shape relationship between HDL-cholesterol and cardiovascular disease. European Journal of Preventive Cardiology, In press, pp.204748731989411. 10.1177/2047487319894114 . hal-02433564

\section{HAL Id: hal-02433564 \\ https: / hal.sorbonne-universite.fr/hal-02433564}

Submitted on 9 Jan 2020

HAL is a multi-disciplinary open access archive for the deposit and dissemination of scientific research documents, whether they are published or not. The documents may come from teaching and research institutions in France or abroad, or from public or private research centers.
L'archive ouverte pluridisciplinaire HAL, est destinée au dépôt et à la diffusion de documents scientifiques de niveau recherche, publiés ou non, émanant des établissements d'enseignement et de recherche français ou étrangers, des laboratoires publics ou privés. 
Free cholesterol transfer to high-density lipoprotein (HDL) upon triglyceride lipolysis underlies the U-shape relationship between HDL-cholesterol and cardiovascular disease

Ma Feng ${ }^{\mathrm{a}, \mathrm{b}}$, Maryam Darabi $^{\mathrm{a}, \mathrm{b}, \mathrm{c}}$, Emilie Tubeuf $^{\mathrm{a}, \mathrm{b}}$, Aurélie Canicio ${ }^{\mathrm{a}, \mathrm{b}, \mathrm{c}}$, Marie Lhomme ${ }^{\mathrm{c}}$, Eric Frisdal ${ }^{\mathrm{a}, \mathrm{b}}$, Sandrine Lanfranchi-Lebreton ${ }^{\mathrm{b}}$, Lucrèce Matheron $^{\mathrm{b}}$, Fabiana Rached ${ }^{\mathrm{a}, \mathrm{b}, \mathrm{d}}$, Maharajah Ponnaiah ${ }^{\mathrm{c}}$, Carlos V. Serrano Jr. ${ }^{\mathrm{d}}$, Raul D. Santos ${ }^{\mathrm{d}}$, Fernando Brites ${ }^{\mathrm{e}}$, Gerard Bolbach ${ }^{\mathrm{b}}$, Emmanuel Gautier ${ }^{\mathrm{a}, \mathrm{b}}$, Thierry Huby ${ }^{\mathrm{a}, \mathrm{b}}$, Alain Carrie $^{\mathrm{a}, \mathrm{b}}$, Eric Bruckert ${ }^{\mathrm{a}, \mathrm{b}, \mathrm{c}, \mathrm{f}}$, Maryse Guerin ${ }^{\mathrm{a}, \mathrm{b}}$, Philippe Couvert ${ }^{\mathrm{a}, \mathrm{b}}$, Philippe Giral ${ }^{\mathrm{a}, \mathrm{b}, \mathrm{c}, \mathrm{f}}$, Philippe Lesnik $^{\mathrm{a}, \mathrm{b}}$, Wilfried Le Goff ${ }^{\mathrm{a}, \mathrm{b}}$, Isabelle Guillas ${ }^{\mathrm{a}, \mathrm{b}}$, Anatol Kontush ${ }^{\mathrm{a}, \mathrm{b}}$ *

${ }^{a}$ National Institute for Health and Medical Research (INSERM) UMR_S 1166, Faculty of Medicine Pitié-

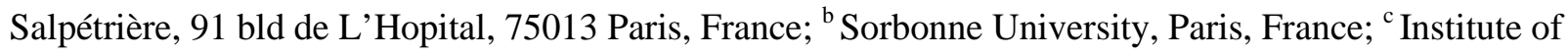
Cardiometabolism and Nutrition (ICAN), Paris, 75013 France; ${ }^{\mathrm{d}}$ Heart Institute-InCor, University of Sao Paulo, Sao Paulo, Brazil; ${ }^{\mathrm{e}}$ Laboratory of Lipids and Atherosclerosis, Department of Clinical Biochemistry, INFIBIOC, University of Buenos Aires, CONICET. Buenos Aires, Argentina; ${ }^{\mathrm{A}}$ AP-HP, Groupe hospitalier Pitié-Salpétrière, Paris, F-75013 France

Part of this work was presented at the $87^{\text {th }}$ European Atherosclerosis Society (EAS) Congress in Maastricht, May 26-29, 2019 as E-poster.

Corresponding author: Anatol Kontush, PhD, Research Director, INSERM Research Unit 1166 - ICAN, Faculty of Medicine Pitié-Salpétrière, Sorbonne University, 91, bd de l'Hôpital, 75013 Paris, France Tel. (33) (1) 407796 33. Fax (33) (1) 407796 45. E-mail anatol.kontush@sorbonne-universite.fr

Word count: 6,533 


\section{Abstract}

Background Low concentrations of high-density lipoprotein-cholesterol (HDL-C) represent a wellestablished cardiovascular risk factor. Paradoxically, extremely high HDL-C levels are equally associated with elevated cardiovascular risk, resulting in the U-shape relationship of HDL-C with cardiovascular disease. Mechanisms underlying this association are presently unknown. We hypothesised that the capacity of HDL to acquire free cholesterol upon triglyceride-rich lipoproteins (TGRL) lipolysis by lipoprotein lipase (LPL) underlies the non-linear relationship between HDL-C and cardiovascular risk. Methods To assess our hypothesis, we developed a novel assay to evaluate the capacity of HDL to acquire free cholesterol (as fluorescent TopFluor ${ }^{\circledR}$ cholesterol) from TRGL upon in vitro lipolysis by LPL.

Results When the assay was applied to several populations markedly differing in plasma HDL-C levels, transfer of free cholesterol was significantly decreased in low HDL-C patients with acute myocardial infarction (-45\%) and Type 2 diabetes (-25\%), and in subjects with extremely high HDL-C of >2.59 mmol/L (>100 mg/dL) (-20\%) vs. healthy normolipidemic controls. When these data were combined and plotted against HDL-C concentrations, an inverse U-shape relationship was observed. Consistent with these findings, animal studies revealed that the capacity of HDL to acquire cholesterol upon lipolysis was reduced in low HDL-C apolipoprotein A-I knock-out mice and was negatively correlated with aortic accumulation of ${ }^{3} \mathrm{H}$-cholesterol after oral gavage, attesting this functional characteristic as a negative metric of postprandial atherosclerosis.

Conclusions Free cholesterol transfer to HDL upon TGRL lipolysis may underlie the U-shape relationship between HDL-C and cardiovascular disease, linking HDL-C to triglyceride metabolism and atherosclerosis.

Keywords lipoprotein lipase, atherosclerosis, postprandial lipid metabolism, apolipoproteins, chylomicrons, HDL function, HDL metabolism, LCAT, lipoprotein metabolism, lipid transfer proteins 


\section{Introduction}

Low levels of plasma high-density lipoprotein-cholesterol (HDL-C) represent a well-established cardiovascular risk factor. ${ }^{1,2}$ However, therapeutic raising of HDL-C on a background of statin treatment does not exert expected beneficial effects on cardiovascular disease (CVD). ${ }^{3}$ Furthermore, large-scale epidemiological studies reveal that both cardiovascular and overall mortality are paradoxically increased at extremely high HDL-C levels, ${ }^{4-6}$ an observation which might account for the negative results of HDLC-raising trials. It has therefore been suggested that it is not HDL-C itself that is causatively related to atheroprotection but rather a cardioprotective HDL function, which cannot always be reliably estimated through the assay of HDL-C. ${ }^{7}$

The major atheroprotective function of HDL is presently thought to involve cholesterol efflux from arterial wall cells with its subsequent transport to the liver for excretion in a process of reverse cholesterol transport (RCT). ${ }^{7}$ Such "HDL flux hypothesis" is based on negative associations of CVD with in vitro measurements of cellular cholesterol efflux from lipid-loaded macrophages. ${ }^{8}$ Cholesterol efflux from tissue macrophages however provides only a small contribution to HDL-C levels if any ${ }^{9}$ and can therefore hardly explain the complex non-linear association between HDL-C and cardiovascular risk. Intravascular HDL metabolism is intimately linked to that of triglyceride-rich lipoproteins (TGRL), a phenomenon which is frequently manifested as a negative correlation between plasma levels of HDL-C and triglycerides. ${ }^{10,11}$ Given that elevated triglyceride levels are increasingly accepted as a cardiovascular risk factor, ${ }^{10,11}$ low HDL-C has been proposed to represent a biomarker of elevated concentrations of atherogenic TGRL remnants, ${ }^{10}$ a clinically attractive concept which however lacks mechanistic insight. In the circulation, HDL and TGRL interact via multiple metabolic pathways a most studied of which includes heteroexchange of core lipids mediated by cholesteryl ester transfer protein (CETP). ${ }^{12}$ A key pathway linking HDL and TGRL involves transfer to HDL of surface remnants generated during TGRL lipolysis by lipoprotein lipase (LPL). ${ }^{13}$ This frequently overlooked process delivers high amounts of free cholesterol (FC) to HDL and constitutes a quantitatively major source of circulating HDL-C, accounting 
for up to $50 \%$ of its variation. ${ }^{14}$ Indeed, intestinal cholesterol secretion within chylomicrons is comparable to net cholesterol efflux from all extra-hepatic tissues combined. ${ }^{15,16}$ Mechanistically, FC acquirement by HDL can be primarily mediated by small-sized complexes containing phospholipid (PL) and apolipoprotein (apo) A-I, the major HDL protein and potent biological detergent. ${ }^{17,18}$ Remarkably, high amounts of apoA-I are synthesised by the intestine to be incorporated into chylomicrons and postprandially transferred to HDL. ${ }^{19}$

To explain the relationship between HDL-C levels and cardiovascular risk, we propose a reverse remnant cholesterol transport (RRT) hypothesis which involves acquirement by HDL of surface remnants of TGRL upon lipolysis with subsequent transport of remnant-derived cholesterol to the liver in a pathway which originates in the intestine with the secretion of apoA-I on chylomicrons followed by their transport to plasma via lymph. As a corollary, we suggest that plasma concentration of HDL-C is an imperfect static measure of cholesterol flux through this dynamic pathway.

To assess this hypothesis, we developed an in vitro fluorescent assay to evaluate the capacity of HDL to acquire FC from TGRL during lipolysis by LPL. The assay was applied to several groups of subjects markedly differing in HDL-C levels. Our findings reveal decreased FC transfer from TGRL to HDL in subjects with both low and extremely high HDL-C, a finding which may link HDL-associated cardiovascular risk to triglyceride metabolism and account for the U-shape relationship of HDL-C and CVD.

\section{Methods}

\section{Study populations}

Four groups of subjects markedly differing in HDL-C levels were studied together with corresponding control groups. Male patients presenting with ST segment elevation acute myocardial infarction (AMI; n=22) were recruited at the Heart Institute-InCor University of Sao Paulo Medical School Hospital (São Paulo, Brazil) before initiation of any treatment within no later than $24 \mathrm{~h}$ of clinical presentation in the Emergency Room. Healthy non-smoking normolipidemic male subjects $(n=8)$ were recruited as controls. 
Treatment-naïve patients with well-controlled Type 2 diabetes (T2D; males and postmenopausal women, n=17) were recruited from the Ramon Carrillo Centre (La Matanza, Buenos Aires, Argentina). Healthy non-smoking normolipidemic subjects $(n=16)$ were recruited from the same geographical area to constitute the control group. Subjects with high (1.81 to $2.59 \mathrm{mmol} / \mathrm{L}$ [70 to $100 \mathrm{mg} / \mathrm{dL}$; $\mathrm{n}=20$ ) and extremely high (>2.59 mmol/L [>100 mg/dL]; n=20) HDL-C levels together with healthy normolipidemic control volunteers ( $\mathrm{n}=20)$ were recruited at the La Pitié-Salpetrière University Hospital (Paris, France).

\section{Measurement of HDL capacity to acquire FC during TGRL lipolysis by LPL}

Given that fluorometric measurements are distinguished by high sensitivity and specificity, introduction in the assay of a fluorescent FC derivative provides excellent means for evaluating FC transfer across lipoproteins. Importantly, selective labelling of TGRL with fluorescent FC implies its absence from HDL at baseline, thereby strongly enhancing its relative enrichment in HDL over time as compared to that of unlabelled FC. We therefore labelled TGRL with fluorescent 23-(dipyrrometheneboron difluoride)-24norcholesterol (Avanti Polar Lipids, Alabaster, AL, USA), also known as TopFluor ${ }^{\circledR}$ cholesterol (TopF) and BODIPY-cholesterol, and evaluated its transfer to HDL during LPL-induced lipolysis.

To isolate TGRL $(\mathrm{d}<1.019 \mathrm{~g} / \mathrm{ml})$, a single-step ultracentrifugation was used. HDL was employed in the form of ultracentrifugally isolated HDL (UC-HDL) or as apoB-depleted plasma. To label human TGRL with TopF, TGRL was added to lipoprotein-deficient plasma (LPDP) at the LPDP:TGRL ratio of 1:100 by volume. The mixture was filtered and TopF was added as a chloroformic solution followed by overnight incubation at $37^{\circ} \mathrm{C}$ under gentle stirring. Labelled TGRL was separated from unbound fluorescent lipid using filtration through a PD-10 Sephadex column. Triglyceride concentration in purified labelled TGRL was measured by photometry and TopF fluorescence was registered at the excitation/emission wavelengths of 500/525 nm using Gemini Microplate Reader (Molecular Devices, USA) to verify the labelling.

To evaluate the transfer of TopF, Tris buffer $(0.4 \mathrm{M}, \mathrm{pH}$ 8) was mixed on ice with required amounts of TopF-labelled TGRL and HDL, and LPL from Pseudomonas sp. or from bovine milk (both from Sigma, 
France) was added to start lipolysis at $37^{\circ} \mathrm{C}$. At the end of the incubation, the reaction mixture was placed on ice and apoB precipitant containing phosphotungstic acid and $\mathrm{MgCl}_{2}$ was added. Following incubation at room temperature and centrifugation, HDL-containing supernatant was aspirated, filtered and transferred to a black microplate for fluorescence reading. Fluorescence of the standard TGRL sample alone was also measured at a triglyceride concentration employed in the assay and fluorescent values measured in HDL were expressed as a percentage of fluorescence of such standard sample. In some experiments, inhibitors for CETP (torcetrapib, $25 \mu \mathrm{M}$ ) and lecithin-cholesterol acyltransferase (LCAT) (iodoacetamide, $750 \mu \mathrm{M}$ ) were used.

To characterise the transfer of TopF to HDL obtained from clinical plasma samples, TGRL and apoBdepleted plasma were used at final concentrations of $0.34 \mathrm{mmol} / \mathrm{L}$ (30 mg triglyceride/dL) and 3.3\%, respectively. Assay conditions (lipoprotein concentrations, incubation time, etc.) were chosen to model those of postprandial TGRL lipolysis in humans. A reference apoB-depleted plasma sample obtained from one healthy normolipidemic control plasma aliquoted and stored at $-80^{\circ} \mathrm{C}$ was included in each series of measurements and all values obtained in clinical samples were normalised to that observed in the reference plasma.

\section{Postprandial aortic cholesterol accumulation in mice}

Postprandial aortic accumulation of FC was evaluated in vivo after gavage with $100 \mu \mathrm{Ci}$ of $\left[{ }^{3} \mathrm{H}\right]-$ cholesterol administrated with olive oil to three groups of mice markedly differing in HDL-C levels, including low-HDL-C apoA-I knock-out mice, high-HDL-C human apoA-I transgenic mice and control wild-type mice. The animals were euthanized $2 \mathrm{~h}$ after the gavage, their aortas removed and weighted, and specific radioactivity measured. HDL capacity to acquire TopF from TGRL during LPL-induced lipolysis was evaluated in apoB-depleted EDTA plasma obtained from every mouse following overnight fast several weeks before the gavage experiment.

A detailed description of the methods is provided in Supplementary Material. 


\section{Results}

HDL isolated by ultracentrifugation or apoB depletion readily acquired TopF upon lipolysis of TopFlabelled TGRL (Fig. 1; Supplemental Figure 1). The transfer was time-dependent and typically saturated after 1-2h, except for the highest concentration of UC-HDL (Fig. 1, A and C). Dose-dependence of the transfer was characterised by an increase with increasing UC-HDL concentrations (Fig. 1, B), while maximal TopF accumulation in HDL isolated by apoB depletion was observed at intermediate concentrations of apoB-depleted plasma, decreasing at higher concentrations (Fig. 1, D). This dosedependence was confirmed by measurements of unlabelled native FC (Supplemental Fig. 2), revealing a correlation coefficient of $0.71(\mathrm{p}<0.001)$ between the two methods and demonstrating the validity of our fluorescence-based approach. In addition, when HDL was re-isolated from the same reaction mixtures by apoB precipitation and by UC, TopF fluorescence readings in such HDL were strongly correlated (r=0.95, $\mathrm{p}=0.014$ ), further validating our approach.

When $\mathrm{V}_{\max }$ and $\mathrm{K}_{\mathrm{d}}$ for the transfer of unlabelled FC were calculated, they revealed maxima at intermediate concentrations of HDL (Fig. 2, A), consistent with the results obtained using fluorescent FC. As a consequence, concentration of apoB-depleted plasma of 3.3\% and UC-HDL concentration of $4 \mathrm{mg}$ protein/dL, the both corresponding to the initial concentration-dependent sections of the dose-dependent curves (Fig. 1, B and D), were chosen to compare individual plasma samples for the capacity of their HDL to acquire FC.

Initial characterisation of the assay revealed good reproducibility of the measurement of TopF transfer to normolipidemic apoB-depleted plasma, with intra- and inter-assay coefficients of variation of $5.1 \%$ and $8.8 \%$, respectively $(\mathrm{n}=10)$. The assay was initially employed to obtain insight into mechanisms of FC transfer from TGRL to HDL during LPL-mediated lipolysis using inhibitors. Both inhibition of CETP using torcetrapib and inhibition of LCAT using iodoacetamide markedly (1.8- and 2.1-fold, respectively) enhanced the accumulation of TopF in UC-HDL (Fig. 2, B). Torcetrapib equally increased the accumulation of TopF when apoB-depleted plasma was used as a source of HDL (from $49 \pm 11 \%$ to 74 
\pm 11 in the absence and presence of $25 \mu \mathrm{M}$ torcetrapib, respectively, in $10 \%$ apoB-depleted plasma; $\mathrm{n}=4$, $\mathrm{p}<0.05$ ), modifying the dose-dependence of the transfer in such a way that its decrease at high concentrations of apoB-depleted plasma was abolished.

The assay was subsequently applied to several groups of patients markedly differing in plasma HDL-C. Patients with AMI featured reduced HDL-C (-31\%, $\mathrm{p}<0.01$; Supplemental Table 1$)$ relative to healthy normolipidemic controls. The capacity of HDL to acquire TopF was decreased in AMI patients by -46\% (controls, $103 \pm 12 \%$ vs. patients, $57 \pm 28 \%$, p<0.001; Fig. 3, A). This decrease was thereby more pronounced as compared to that in HDL-C levels. The capacity of HDL to acquire TopF was positively correlated with HDL-C in this low HDL-C group $(r=0.49, \mathrm{p}<0.05)$. By contrast, accumulation of TopF in UC-HDL isolated from AMI plasma was similar to that observed in UC-HDL from controls when the lipoproteins were compared on a protein concentration basis (Supplemental Fig. 3, A). To evaluate whether between-group differences were specifically related to the transfer of fluorescent cholesterol, we measured the accumulation of unlabelled native FC in HDL from a subpopulation of AMI and control subjects. The transfer of FC was strongly (6.5-fold, $\mathrm{p}<0.01$ ) reduced in AMI relative to control HDL (Supplemental Fig. 3, B) and was correlated with measurements of TopF-cholesterol ( $r=0.73, \mathrm{p}=0.005$ ). Treatment-naïve patients with well-equilibrated T2D, an established cardiovascular risk factor, were characterised by a typical lipid profile involving reduced HDL-C $(-21 \%, \mathrm{p}<0.05)$ and elevated triglycerides (+94\%, $\mathrm{p}<0.05$ ) as compared to healthy normolipidemic controls (Supplemental Table 2). The capacity of HDL to acquire FC was decreased in T2D patients by $-24 \%$ (controls, $96 \pm 28 \%$ vs. patients, 72 $\pm 33 \%, \mathrm{p}<0.05$; Fig. 3, A). This decrease was similar to that in HDL-C levels. As compared to healthy, normolipidemic controls, subjects with high HDL-C levels (from 1.81 to 2.59 mmol/L [70 to $100 \mathrm{mg} / \mathrm{dL}]$ ) were characterised by elevated HDL-C (+34\%, $\mathrm{p}<0.001$; Supplemental Table 3). Despite such marked increase, the capacity of HDL to acquire FC did not differ between the groups (controls, $97 \pm 22 \%$ vs. patients, $94 \pm 33 \%$, Fig. 3, A), thereby diverging from the HDL-C assay. In subjects with extremely high HDL-C concentrations (>2.59 mmol/L [>100 mg/dL]), HDL-C was elevated almost 2-fold (+92\%) relative to controls (Supplemental Table 4). Despite such pronounced 
increase, the capacity of HDL to acquire FC was diminished by $-20 \%$ in this group (controls, $101 \pm 20 \%$ vs. patients, 81 $\pm 27 \%$, $<<0.05$; Fig. 3, A), further diverging from HDL-C. As a result, no correlation between HDL capacity to acquire TopF and plasma HDL-C was observed across all the populations studied $(\mathrm{r}=0.10, \mathrm{p}=0.29 ; \mathrm{n}=123)$. Such absence of linear correlation was related to a complex non-linear dependence between TopF transfer and HDL-C observed across both individual subjects (Fig. 3, B) and studied populations (Fig. 3, C), which resembled an inverse U-shape. It is of note that no difference in the capacity of HDL to acquire TopF was observed between the four control groups, validating our study design (Fig. 3, A).

To evaluate potential determinants of the reduced FC transfer to HDL, plasma activities of CETP and LCAT were assessed in the patients using fluorometric kits. The only significant difference observed relative to controls involved reduced CETP activity in AMI patients (-50\%, p<0.01; Supplemental Fig. 4). Given that subjects with low and high HDL-C levels greatly differ in their HDL particle profile with large HDL prevailing at high HDL-C, ${ }^{20}$ we evaluated as to whether HDL particle subpopulations differed in their capacity to acquire FC upon TGRL lipolysis by LPL. We found that large, light HDL2 particles were up to 3.8-fold less efficient acceptors for TopF relative to small, dense HDL3 at a fixed protein concentration of $4 \mathrm{mg} / \mathrm{dL}$ (Supplemental Fig. 5), equivalent to 2.8-fold per particle difference. The relationship of the assay with CVD was initially evaluated on the basis of published mortality data. When cardiovascular and overall mortality data obtained from large-scale CANHEART HDL study ${ }^{4}$ and Copenhagen City Heart Study ${ }^{6}$ were recalculated for the mean HDL-C levels observed in the five populations studied by us (AMI, T2D, subjects with high and extremely high HDL-C, and pooled controls) and plotted against HDL-C, a U-shape relationship was observed as reported ${ }^{4,6}$ (Supplemental Fig. 6, A). In striking contrast, when HDL-C was replaced by the mean values of HDL capacity to acquire TopF, linear negative relationships with both cardiovascular and overall mortality were obtained $\left(\mathrm{r}^{2}\right.$ from 0.79 to 0.97 , p from 0.043 to 0.002 ), indicative of the significant relationship of mortality with the assay outcome (Supplemental Fig. 6, B). 
Pathophysiological relevance of the assay was further evaluated in vivo using murine models displaying marked differences in circulating HDL-C levels, specifically wild-type mice, dyslipidemic low HDL-C apoA-I -/- mice and high HDL-C mice transgenic for human apoA-I. As expected, HDL-C concentrations were reduced by $-82 \%$ in apoA-I knock-out mice and elevated by $+72 \%$ in human apoA-I transgenic animals (Fig. 4, A). Expectedly, CETP activity was nearly absent from plasma of all mice and LCAT activity was greatly reduced relative to human plasma (Supplemental Fig. 7), with apoA-I knock-out animals featuring the lowest LCAT activity.

The capacity of HDL to acquire FC measured upon TGRL lipolysis by LPL in vitro using TopF was reduced by $-38 \%(\mathrm{p}<0.01)$ in apoA-I knock-out mice but was not modified in human apoA-I transgenic animals (Fig. 4, B). When all the mice received an oral gavage of ${ }^{3} \mathrm{H}$-cholesterol together with olive oil, postprandial accumulation of ${ }^{3} \mathrm{H}$-cholesterol in the aorta was significantly (6.1-fold) enhanced $2 \mathrm{~h}$ after the gavage in apoA-I knock-out relative to wild-type mice, while no difference between wild-type and human apoA-I transgenic mice was found (Fig. 4, C). As a consequence, the capacity of HDL to acquire FC in vitro was significantly and negatively correlated with the aortic accumulation of ${ }^{3} \mathrm{H}$-cholesterol in vivo across all mice, attesting this functional parameter as a negative metric of postprandial atherosclerosis (Fig. 4, D).

\section{Discussion}

In the present study, we evaluated our RRT hypothesis that FC transfer to HDL upon TGRL lipolysis by LPL underlies the complex relationship between HDL-C levels and cardiovascular risk. We provide evidence, using human subjects and animal models markedly differing in HDL-C concentrations, that this process links triglyceride metabolism to atherosclerosis and may account for the relationship between HDL-C and CVD.

To assess our hypothesis, we developed an original method to evaluate in vitro FC transfer from TGRL to HDL upon LPL-mediated lipolysis of TGRL labelled with TopF, a fluorescent derivative of FC. Earlier studies of material exchange between TGRL and HDL revealed that multiple lipid and protein 
components, including FC, PL and apolipoproteins, were transferred from TGRL to HDL upon LPLmediated lipolysis. ${ }^{13,14,21,22}$ Mechanisms of such exchange are defined by the removal of excess surface fragments from TGRL, which cannot adapt the structure of remnant particles possessing reduced hydrophobic core. Formation of surface TGRL remnants possibly involves spontaneous organisation of excessive surface lipids separated from TGRL into low-energy complexes which resemble micelles or liposomes. $^{23}$

ApoA-I, the major HDL protein present in high amounts on chylomicrons, ${ }^{19}$ can play a central role in this process as a result of its distinct lipid-binding properties. ${ }^{17}{ }^{18}$ Indeed, apoA-I avidly interacts with minimal amounts of lipids present in aqueous solutions, transforming them into highly organised lipoprotein complexes in the HDL density range. ${ }^{17,18}$ While apoA-I can solubilise excess surface lipids released from TGRL upon lipolysis, HDL PL may provide lipid surface required for their absorption. ${ }^{21,23,24}$ Other structural HDL apolipoproteins, including apoA-II, apoE and apoM, can equally be expected to participate in lipid transfer during lipolysis, consistent with the presence of FC acceptor activity in HDL from apoA-I -/- mice.

As lipid transfer proteins play key roles in exchanging lipids across lipoproteins, these proteins may actively participate in the transfer of surface lipids upon LPL-mediated lipolysis. To assess this possibility, we employed torcetrapib, a specific CETP inhibitor, and observed enhanced transfer of FC upon inhibition. Such inhibitory role of CETP towards cholesterol removal from TGRL can be explained by its capacity to transfer cholesterol, in a form of cholesteryl ester (CE), from HDL back to TGRL. ${ }^{12}$ Indeed, HDL represents a major source of CE in human plasma, reflecting preferential association of LCAT with HDL. ${ }^{16}$ When FC is transferred from TGRL to HDL and esterified under the action of LCAT, a molecule of CE formed becomes a substrate for CETP and can be readily transferred back to TGRL in exchange for a molecule of TGRL-derived triglyceride. This mechanism can be operative for TopF as it is efficiently esterified by LCAT and as its ester is transferred by CETP in vitro. ${ }^{25,26}$ If this mechanism is true and LCAT and CETP act along the same pathway of cholesterol removal from HDL, then inhibition of LCAT should enhance LPL-induced accumulation of TGRL-derived FC in HDL. 
Consistent with this conclusion, we did observe accelerated accumulation of TopF upon LCAT inhibition by iodoacetamide, suggesting a multi-step mechanism of FC movement between HDL and TGRL upon LPL-induced lipolysis, which involves acquirement of FC by HDL with its subsequent esterification by LCAT followed by CETP-mediated exchange of the generated CE for triglyceride present in TGRL. Importantly, kinetic data reveal that esterification of FC by LCAT occurs more slowly than both facilitated diffusion of CE mediated by CETP and FC transfer across lipoproteins, ${ }^{16,27,28}$ thereby constituting a rate-limiting step in the exchange of cholesterol between TGRL and HDL. Such CE transfer from HDL to TGRL might therefore be negligibly low at low concentrations of LCAT (and low concentrations of HDL, as LCAT is exclusively carried by HDL under our experimental conditions) but might greatly increase at high HDL concentrations, decreasing net cholesterol accumulation in HDL. Reduction of FC concentration gradient between TGRL and HDL occurring at high HDL concentrations may additionally contribute to the latter effect. Consistent with this notion, the dose-dependence of FC transfer was characterised by direct relationship with HDL concentration at low concentrations of both UC-HDL and apoB-depleted plasma, most likely reflecting the presence in the assay of increasing amounts of acceptor HDL particles for TGRL-derived FC. However, such direct relationship evolved into an inversed dose-dependence at high concentrations of HDL when apoB-depleted plasma (possessing high CETP activity) was employed as the HDL source. Interestingly, the inverse relationship was not observed in apoB-depleted plasma in the presence of torcetrapib, or in UC-HDL (containing only minor amounts of CETP following $\mathrm{UC}^{29}$ ). In the latter experiment, the transfer of FC was directly related to HDL concentration throughout the whole concentration range studied, potentially reflecting low CETP activity in the samples.

Clinical value of the assay was assessed in four populations markedly differing in plasma HDL-C. The transfer of FC to HDL was reduced in low HDL-C patients with AMI, low HDL-C patients with T2D and subjects with extremely high HDL-C levels of $>100 \mathrm{mg} / \mathrm{dL}$ relative to healthy normolipidemic controls. When these data were plotted against individual HDL-C levels in all subjects, or against mean HDL-C levels in the groups, inverse U-shape relationships were observed which mirrored the U-shape relationship 
of overall and cardiovascular mortality with HDL-C concentrations recently reported in large-scale epidemiological studies. ${ }^{4-6}$ As a consequence, the relationships of FC transfer to HDL with cardiovascular and overall mortality obtained from these studies were linear and negative, in clear contrast to the U-shape relationships observed for HDL-C. Albeit circumstantial, these data suggest that FC transfer to HDL upon TGRL lipolysis by LPL may underlie the U-shape relationship between HDL-C and CVD.

Importantly, FC transfer was unchanged in subjects with high HDL-C of 70 to $100 \mathrm{mg} / \mathrm{dL}$ and was only diminished at extremely high HDL-C levels of $>100 \mathrm{mg} / \mathrm{dL}$. It is worth noting that the high HDL-C groups expectedly featured a prevalence of female over male subjects, while the AMI group was only composed of males, rising a question of the role of gender for the observed differences. Each of the studied groups was however compared to a matched control group and no difference in the TopF transfer was observed between the four control groups despite their differences in the sex ratio, thereby arguing against the role of gender in the between-group effects observed by us.

According to the dose-dependences of HDL capacity to acquire FC from TGRL upon lipolysis, the decreases in this metric observed in low HDL-C patients with AMI and T2D may straightforwardly reflect low concentrations of acceptor HDL particles. Indeed, no difference in FC transfer to AMI and control HDL was found when the lipoproteins were compared on a protein concentration basis. Other metabolic alterations potentially underlying decreased FC transfer in both low and extremely high HDL-C subjects involve elevated CETP and/or LCAT activities, which were however not detected by us. Altered HDL particle profile typical of extremely high HDL-C states includes highly elevated levels of large, lipid-rich HDL and reduced concentrations of small, lipid-poor HDL particles. ${ }^{20,30}$ As our data document superior capacity of small vs. large HDL to acquire FC upon TGRL lipolysis (which is consistent with the superiority of such particles to efflux cellular cholesterol and to perform other biological functions ${ }^{31}$ ), the decrease in this metric at extremely high HDL-C might reflect such alterations in the HDL particle profile. To establish a direct link between lipolytic FC transfer from TGRL to HDL and development of CVD, we studied postprandial movement of radiolabelled cholesterol in three groups of mice markedly differing in HDL-C. Small amounts of $\left[{ }^{3} \mathrm{H}\right]$-cholesterol were consistently found in murine aortic tissue $2 \mathrm{~h}$ following 
oral gavage with this compound. Such aortic accumulation of $\left[{ }^{3} \mathrm{H}\right]$-cholesterol was inversely associated with HDL-C concentrations, being highly elevated in low HDL-C apoA-I knock-out animals. In parallel, the capacity of murine HDL to acquire FC upon TGRL lipolysis by LPL was reduced, resulting in its negative correlation with the aortic accumulation of $\left[{ }^{3} \mathrm{H}\right]$-cholesterol. These data additionally attest FC transfer to HDL upon TGRL lipolysis by LPL as a negative metric of CVD and directly link HDL metabolism to postprandial atherosclerosis. Interestingly, FC transfer was not compromised in high HDLC apoA-I transgenic mice, in contrast to our observations of reduced FC transfer in human subjects with extremely high HDL-C, potentially reflecting low CETP and LCAT activities in mice.

Together, our data provide evidence that HDL capacity to acquire FC upon TGRL lipolysis by LPL in vitro, measured using our novel assay, may represent a superior biomarker of cardiovascular risk as compared to plasma HDL-C levels presently employed in clinical practice. The assay is simple, rapid, does not require sophisticated equipment and can be easily run in clinical routine. Moreover, these data support our RRT hypothesis to explain the U-shape association between HDL-C levels and CVD by intravascular FC transfer from TGRL to HDL in a pathway that can be essential for triglyceride metabolism and energy production. We propose that plasma concentration of HDL-C is an imperfect static measure of cholesterol flux through this dynamic pathway, which is directly associated with the efficacy of cholesterol removal from the circulation only at low-to-normal HDL-C. Importantly, HDL exerts a plethora of other biological activities, including its capacity to efflux cholesterol from peripheral cells, to protect LDL from oxidative modification, to reduce production of pro-inflammatory phospholipids and to beneficially impact glucose metabolism. ${ }^{32,33}$ None of these wellstudied activities is however known to be reduced at both low and extremely high concentrations of HDL and can thereby account for the complex non-linear relationship between HDL-C levels and CVD. We conclude that this relationship primarily reflects the lipid acceptor role of HDL in the lipolysis. 


\section{Acknowledgements}

Excellent technical assistance from Ms Julie Tomczyk, Mr Henri Voedts, Mr. Narcisse Medadje and Mr Jean Brerault is deeply appreciated.

\section{Funding}

These studies were supported by National Institute for Health and Medical Research (INSERM; Paris, France). We gratefully acknowledge further support from the University of Pierre and Marie Curie (UPMC; Paris, France), ICAN (Paris, France), China Scholarship Council (CSC, China), CAPES (Brazil), FAPESP (Brazil), and a collaborative grant from CONICET (Buenos Aires, Argentina), University of Buenos Aires (UBACyT CB23), Craveri Foundation and CONICET (PIP 516).

\section{Conflict of Interest}

Dr. Kontush reports grants from CSL and Pfizer, personal fees from American Heart Association, National Lipid Association, Sanofi and European Society of Cardiology, outside the submitted work. In addition, Dr. Kontush has a patent EP17306042 entitled “Novel assay of HDL function”.

\section{Authors' Contributions}

Designing research studies: MF, MD, ML, LM, EF, FR, CVS, RDS, FB, GB, EG, TH, AC, EB, MG, PC, PG, PL, WLG, IG, AK

Recruiting study subjects: FR, CVS, RDS, FB, EB, MG, PC, PG

Conducting experiments: MF, MD, ET, AC, SL, IG, AK

Acquiring data: MF, MD, ET, AC, ML, LM, SL, FB, CVD, FB, AC, EB, MG, PC, PG, IG, AK

Analyzing data: MF, MD, MP, IG, AK

Writing the manuscript: MF, AK 


\section{References}

1. Kontush A and Chapman MJ. High-Density Lipoproteins: Structure, Metabolism, Function and Therapeutics. New York: Wiley \& Sons, 2012, p.648.

2. Luscher TF, Landmesser U, von Eckardstein A and Fogelman AM. High-density lipoprotein: vascular protective effects, dysfunction, and potential as therapeutic target. Circ Res. 2014; 114: 171-82.

3. Keene D, Price C, Shun-Shin MJ and Francis DP. Effect on cardiovascular risk of high density lipoprotein targeted drug treatments niacin, fibrates, and CETP inhibitors: meta-analysis of randomised controlled trials including 117,411 patients. BMJ. 2014; 349: g4379.

4. Ko DT, Alter DA, Guo H, et al. High-Density Lipoprotein Cholesterol and Cause-Specific Mortality in Individuals Without Previous Cardiovascular Conditions: The CANHEART Study. $J$ Am Coll Cardiol. 2016; 68: 2073-83.

5. Hirata A, Okamura T, Sugiyama D, et al. The Relationship between Very High Levels of Serum High-Density Lipoprotein Cholesterol and Cause-Specific Mortality in a 20-Year FollowUp Study of Japanese General Population. J Atheroscler Thromb. 2016; 23: 800-9.

6. Madsen CM, Varbo A and Nordestgaard BG. Extreme high high-density lipoprotein cholesterol is paradoxically associated with high mortality in men and women: two prospective cohort studies. Eur Heart J. 2017; 38: 2478-86.

7. Rader DJ and Tall AR. The not-so-simple HDL story: Is it time to revise the HDL cholesterol hypothesis? Nat Med. 2012; 18: 1344-6.

8. Bhatt A and Rohatgi A. HDL Cholesterol Efflux Capacity: Cardiovascular Risk Factor and Potential Therapeutic Target. Curr Atheroscler Rep. 2016; 18: 2. 
9. Rosenson RS, Brewer HB, Jr., Davidson WS, et al. Cholesterol efflux and atheroprotection: advancing the concept of reverse cholesterol transport. Circulation. 2012; 125: 1905-19.

10. Nordestgaard BG and Varbo A. Triglycerides and cardiovascular disease. Lancet. 2014; 384: 626-35.

11. Rosenson RS, Davidson MH, Hirsh BJ, Kathiresan S and Gaudet D. Genetics and causality of triglyceride-rich lipoproteins in atherosclerotic cardiovascular disease. J Am Coll Cardiol. 2014; 64: 2525-40.

12. Chapman MJ, Le Goff W, Guerin M and Kontush A. Cholesteryl ester transfer protein: at the heart of the action of lipid-modulating therapy with statins, fibrates, niacin, and cholesteryl ester transfer protein inhibitors. Eur Heart J. 2010; 31: 149-64.

13. Nikkila EA, Taskinen MR and Sane T. Plasma high-density lipoprotein concentration and subfraction distribution in relation to triglyceride metabolism. Am Heart J. 1987; 113: 543-8.

14. Magill P, Rao SN, Miller NE, et al. Relationships between the metabolism of high-density and very-low-density lipoproteins in man: studies of apolipoprotein kinetics and adipose tissue lipoprotein lipase activity. Eur J Clin Invest. 1982; 12: 113-20.

15. Ros E. Intestinal absorption of triglyceride and cholesterol. Dietary and pharmacological inhibition to reduce cardiovascular risk. Atherosclerosis. 2000; 151: 357-79.

16. Schwartz CC, VandenBroek JM and Cooper PS. Lipoprotein cholesteryl ester production, transfer, and output in vivo in humans. J Lipid Res. 2004; 45: 1594-607.

17. Pownall HJ and Ehnholm C. The unique role of apolipoprotein A-I in HDL remodeling and metabolism. Curr Opin Lipidol. 2006; 17: 209-13.

18. Huang R, Silva RA, Jerome WG, et al. Apolipoprotein A-I structural organization in highdensity lipoproteins isolated from human plasma. Nat Struct Mol Biol. 2011; 18: 416-22. 
19. Bisgaier CL and Glickman RM. Intestinal synthesis, secretion, and transport of lipoproteins. Annu Rev Physiol. 1983; 45: 625-36.

20. Kontush A, de Faria EC, Chantepie S and Chapman MJ. Antioxidative activity of HDL particle subspecies is impaired in hyperalphalipoproteinemia: relevance of enzymatic and physicochemical properties. Arterioscler Thromb Vasc Biol. 2004; 24: 526-33.

21. Chung BH and Dashti N. Lipolytic remnants of human VLDL produced in vitro. Effect of HDL levels in the lipolysis mixtures on the apoCs to apoE ratio and metabolic properties of VLDL core remnants. J Lipid Res. 2000; 41: 285-97.

22. Rutledge JC, Mullick AE, Gardner G and Goldberg IJ. Direct visualization of lipid deposition and reverse lipid transport in a perfused artery - Roles of VLDL and HDL. Circulation Research. 2000; 86: 768-73.

23. Chung BH, Tallis G, Yalamoori V, Anantharamaiah GM and Segrest JP. Liposome-like particles isolated from human atherosclerotic plaques are structurally and compositionally similar to surface remnants of triglyceride-rich lipoproteins. Arterioscler Thromb. 1994; 14: 622-35.

24. Schaefer EJ, Wetzel MG, Bengtsson G, Scow RO, Brewer HB, Jr. and Olivecrona T. Transfer of human lymph chylomicron constituents to other lipoprotein density fractions during in vitro lipolysis. J Lipid Res. 1982; 23: 1259-73.

25. Sakurai T, Sakurai A, Vaisman BL, et al. Development of a novel fluorescent activity assay for lecithin:cholesterol acyltransferase. Ann Clin Biochem. 2017: 4563217733285.

26. Bisgaier CL, Minton LL, Essenburg AD, White A and Homan R. Use of fluorescent cholesteryl ester microemulsions in cholesteryl ester transfer protein assays. J Lipid Res. 1993; 34: 1625-34.

27. Kosek AB, Durbin D and Jonas A. Binding affinity and reactivity of lecithin cholesterol acyltransferase with native lipoproteins. Biochem Biophys Res Commun. 1999; 258: 548-51. 
28. Paromov VM and Morton RE. Lipid transfer inhibitor protein defines the participation of high density lipoprotein subfractions in lipid transfer reactions mediated by cholesterol ester transfer protein (CETP). J Biol Chem. 2003; 278: 40859-66. Epub 2003 Aug 7.

29. Vaisar T, Pennathur S, Green PS, et al. Shotgun proteomics implicates protease inhibition and complement activation in the antiinflammatory properties of HDL. J Clin Invest. 2007; 117: 746-56.

30. Asztalos BF, Horvath KV, Kajinami K, et al. Apolipoprotein composition of HDL in cholesteryl ester transfer protein deficiency. J Lipid Res. 2004; 45: 448-55.

31. Camont L, Lhomme M, Rached F, et al. Small, dense high-density lipoprotein-3 particles are enriched in negatively charged phospholipids: relevance to cellular cholesterol efflux, antioxidative, antithrombotic, anti-inflammatory, and antiapoptotic functionalities. Arterioscler Thromb Vasc Biol. 2013; 33: 2715-23.

32. Allard-Ratick MP, Kindya BR, Khambhati J, et al. HDL: Fact, fiction, or function? HDL cholesterol and cardiovascular risk. European journal of preventive cardiology. 2019: 2047487319848214.

33. Richart AL, Heywood SE, Siebel AL and Kingwell BA. High-density lipoprotein and cardiac glucose metabolism: Implications for management of acute coronary syndromes. European journal of preventive cardiology. 2018; 25: 273-5. 


\section{Figure Legends}

Figure 1. Kinetics and dose-dependences of the transfer of fluorescent FC from TGRL to HDL during LPL-induced lipolysis. HDL was isolated from normolipidemic EDTA plasma by density gradient ultracentrifugation (A, B) or by apoB depletion (C, D) and incubated for up to $3 \mathrm{~h}$ at $37^{\circ} \mathrm{C}$ with LPL (190 U/ml) and TGRL (30 mg TG/dL) labelled with TopF, at a final concentration of 2.5 to $20 \mathrm{mg}$ HDL protein/dL (A, B) or at a final dilution of apoB-depleted plasma of 2.5 to $20 \% \mathrm{v} / \mathrm{v}$ (C, D), respectively. At the end of the incubation, HDL was separated from TGRL by apoB depletion and TopF fluorescence was measured in HDL at the excitation/emission wavelengths of 500/525 nm. Mean values of four to six independent experiments performed with four individual HDL samples are shown. Please note that means with SDs are shown in Supplemental Figure 1 for the sake of clarity. ${ }^{* * *} \mathrm{p}<0.001$, ${ }^{* *} \mathrm{p}<0.01,{ }^{*} \mathrm{p}<0.05$ vs. $0 \mathrm{~h} ;{ }^{\dagger \dagger} \mathrm{p}<0.01,{ }^{\dagger} \mathrm{p}<0.05$ vs. $1 \mathrm{~h}(\mathrm{~A}, \mathrm{C}) ;{ }^{* * *} \mathrm{p}<0.001,{ }^{* *} \mathrm{p}<0.01,{ }^{*} \mathrm{p}<0.05$ vs. $0 \mathrm{mg} / \mathrm{dL}$; ${ }^{+\dagger} \mathrm{p}<0.01,{ }^{\dagger} \mathrm{p}<0.05$ vs. $2.5 \mathrm{mg} / \mathrm{dL} ;{ }^{\ddagger} \mathrm{p}<0.05$ vs. $5 \mathrm{mg} / \mathrm{dL}$ (B); ${ }^{* *} \mathrm{p}<0.01,{ }^{*} \mathrm{p}<0.05$ vs. $0 \% ;{ }^{\dagger} \mathrm{p}<0.05$ vs. $2.5 \%$; ${ }^{\ddagger} \mathrm{p}<0.05$ vs. 5\% (D). TopF, TopFluor ${ }^{\circledR}$ cholesterol.

Figure 2. Kinetic parameters of FC transfer from TGRL to HDL during LPL-induced lipolysis and effects of inhibitors. HDL was isolated from normolipidemic EDTA plasma by apoB depletion and incubated for $2 \mathrm{~h}$ at $37^{\circ} \mathrm{C}$ with LPL (190 U/ml) and TGRL (7.5, 15, 30 and $60 \mathrm{mg}$ TG/dL) at a final dilution of apoB-depleted plasma of 2.5, 5, 10 and 20\% v/v. At the end of the incubation, HDL was separated from TGRL by apoB depletion, FC was measured in HDL using the Amplex Red kit and $\mathrm{K}_{\mathrm{d}}$ and $\mathrm{V}_{\max }$. were calculated according to Lineweaver - Burk. To evaluate the effects of inhibitors, UC-HDL (4 mg protein/dL) isolated from normolipidemic EDTA plasma was incubated for 2h with LPL (190 U/ml) and TopF-labelled TGRL (30 mg triglyceride/dL) in the absence or presence of torcetrapib (25 $\mu \mathrm{M}$ ) or iodoacetamide $(750 \mu \mathrm{M})$. Data from three independent experiments performed with three individual HDL samples are shown; ${ }^{*} \mathrm{p}<0.05$ vs. $0 \mathrm{~h} ;{ }^{\dagger} \mathrm{p}<0.05$ vs. $2 \mathrm{~h}$ incubation in the absence of the inhibitors. TopF, TopFluor® cholesterol. 
Figure 3. Transfer of fluorescent FC from TGRL during LPL-induced lipolysis to HDL isolated from patients with AMI (n=22), T2D (n=17), high HDL-C (n=20) and extremely high HDL-C (n=20). HDL was isolated from EDTA plasma by apoB depletion and incubated for $2 \mathrm{~h}$ at $37^{\circ} \mathrm{C}$ with LPL (190 U/ml) and TopF-labelled TGRL (30 mg triglyceride/dL) at a final concentration of apoB-depleted plasma of 3.3\% v/v. At the end of the incubation, HDL was separated from TGRL by apoB depletion and TopF fluorescence was measured at the excitation/emission wavelengths of 500/525 nm. The horizontal lines depict mean values in each group; ${ }^{* * *} \mathrm{p}<0.001,{ }^{*} \mathrm{p}<0.05$ vs. HDL from corresponding healthy normolipidemic controls (A). The relationship between TopF transfer and plasma HDL-C levels was plotted using least-square data fitting for all individual subjects studied (B) as well as for the means \pm SDs of TopF transfer and HDL-C in each group using ggplot2 (C). In the latter graph, all controls were combined to form a single control group. TopF, TopFluor ${ }^{\circledR}$ cholesterol; H-HDL, high HDL-C; EH-HDL, extremely high HDL-C.

Figure 4. Relationships of plasma HDL-C levels and HDL capacity to acquire fluorescent FC from TGRL upon LPL-induced lipolysis with postprandial aortic cholesterol accumulation in mice. HDLC concentrations (A) and capacity of HDL (as 3.3\% apoB-depleted EDTA plasma; B) to acquire TopF from TGRL (30 mg triglyceride/dL) during LPL-induced lipolysis were measured in human apoA-I transgenic (hapoA-I tg; $\mathrm{n}=6$ to 12), apoA-I knock-out ( $\mathrm{n}=6$ to 10) and control wild-type (wt; $\mathrm{n}=7$ to 14) mice. Postprandial aortic accumulation of cholesterol (C) was evaluated in vivo after gavage with 100 $\mu \mathrm{Ci}$ of $\left[{ }^{3} \mathrm{H}\right]$-cholesterol administrated with olive oil (100 $\left.\mu \mathrm{l}\right)$. The animals were euthanized $2 \mathrm{~h}$ after the gavage, their aortas removed and specific radioactivity measured per wet tissue weight. The relationship between postprandial aortic accumulation of $\left[{ }^{3} \mathrm{H}\right]$-cholesterol in vivo and capacity of HDL to acquire TopF from TGRL during LPL-mediated lipolysis in vitro was plotted using exponential data fitting (D). The horizontal lines depict mean values in each group; ${ }^{* * *} \mathrm{p}<0.001,{ }^{* *} \mathrm{p}<0.01$ vs. wild-type mice; ${ }^{+\dagger} \mathrm{p}<0.001,{ }^{\dagger \dagger} \mathrm{p}<0.05,{ }^{\dagger} \mathrm{p}<0.05$ vs. human apoA-I transgenic mice. HDL-C, HDL-cholesterol; TopF, TopFluor® cholesterol. 

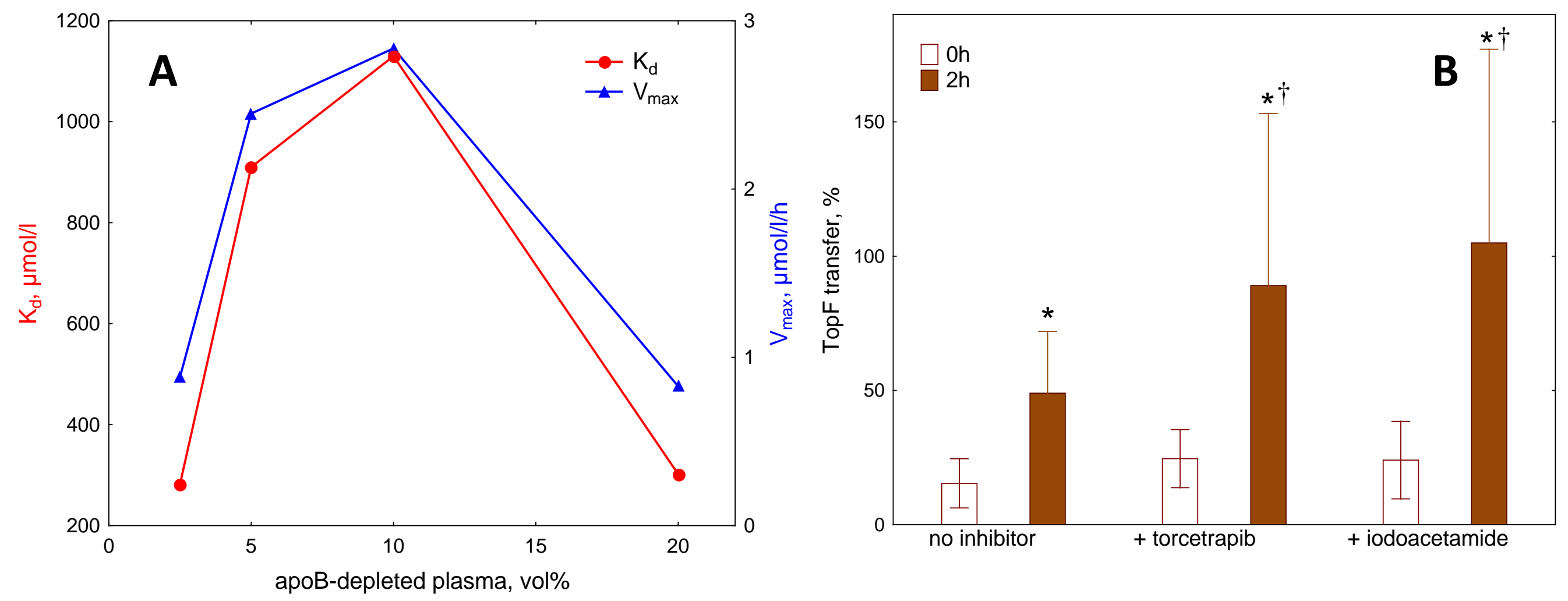

Figure 2 

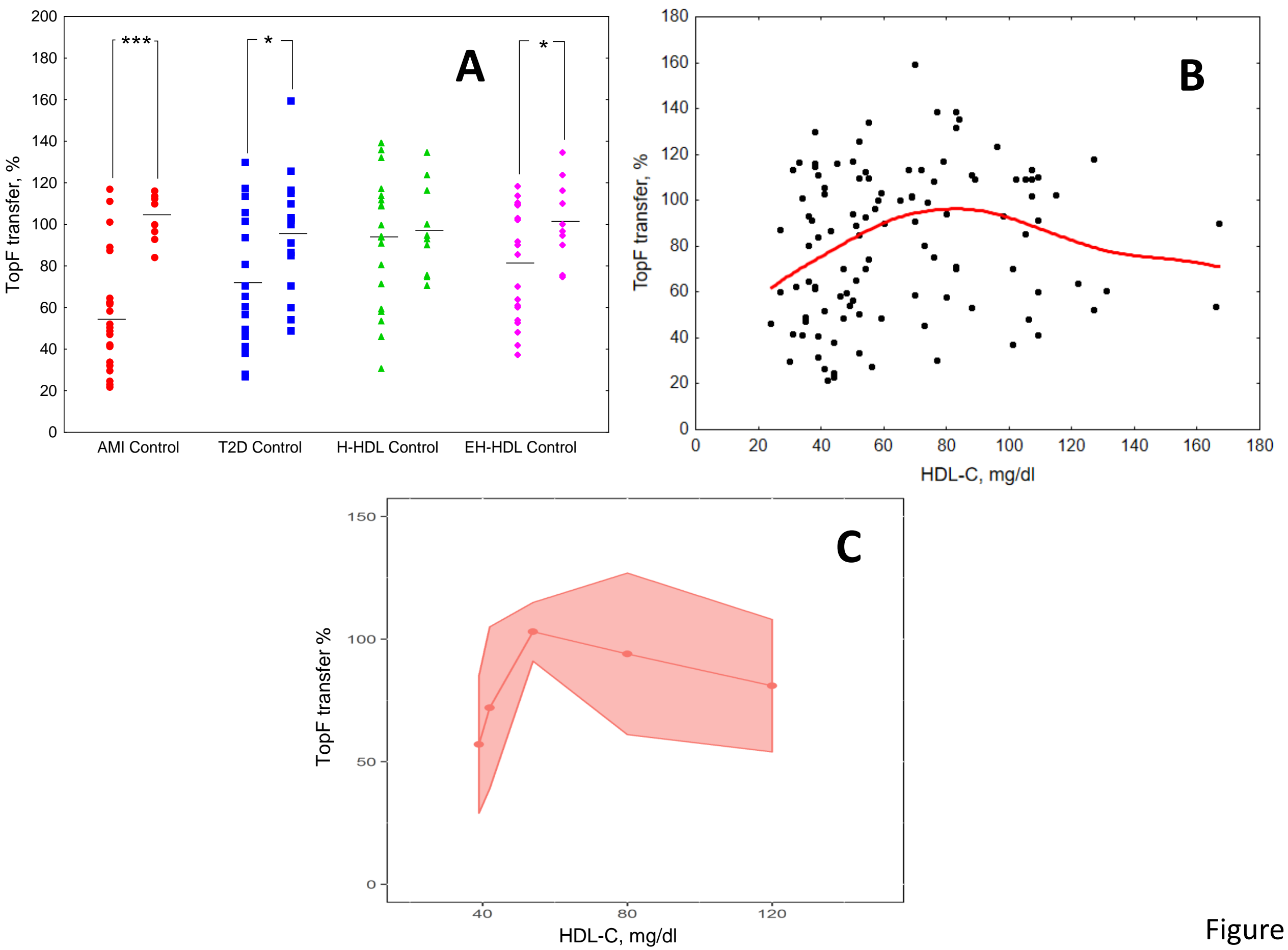

Figure 3 

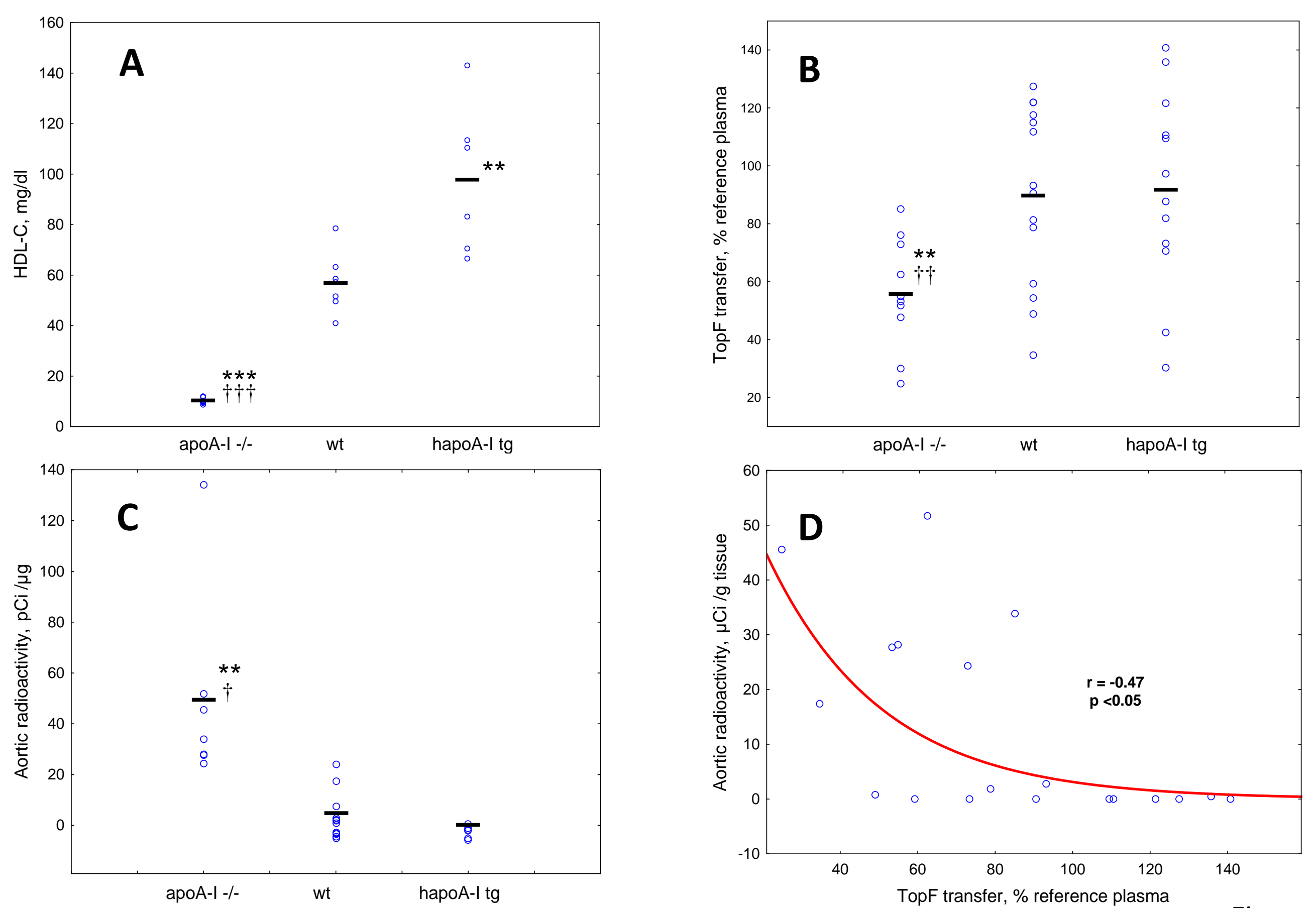

Figure 4 


\section{Supplementary Material}

Free cholesterol transfer to high-density lipoprotein (HDL) upon triglyceride lipolysis underlies the U-shape relationship between HDL-cholesterol and cardiovascular disease

Ma Feng ${ }^{\mathrm{a}, \mathrm{b}}$, Maryam Darabi ${ }^{\mathrm{a}, \mathrm{b}, \mathrm{c}}$, Emilie Tubeuf ${ }^{\mathrm{a}, \mathrm{b}}$, Aurélie Canicio ${ }^{\mathrm{a}, \mathrm{b}, \mathrm{c}}$, Marie Lhomme ${ }^{\mathrm{c}}$, Lucrèce Matheron $^{\mathrm{b}}$, Eric Frisdal ${ }^{\mathrm{a}, \mathrm{b}}$, Sandrine Lanfranchi-Lebreton ${ }^{\mathrm{b}}$, Fabiana Rached ${ }^{\mathrm{a}, \mathrm{b}, \mathrm{d}}$, Maharajah Ponnaiah ${ }^{\mathrm{c}}$, Carlos V. Serrano Jr. ${ }^{\mathrm{d}}$, Raul D. Santos ${ }^{\mathrm{d}}$, Fernando Brites ${ }^{\mathrm{e}}$, Gerard Bolbach ${ }^{\mathrm{b}}$, Emmanuel Gautier ${ }^{\mathrm{a}, \mathrm{b}}$, Thierry Huby ${ }^{\mathrm{a}, \mathrm{b}}$, Alain Carrie ${ }^{\mathrm{a}, \mathrm{b}}$, Eric Bruckert ${ }^{\mathrm{a}, \mathrm{b}, \mathrm{c}, \mathrm{f}}$, Maryse Guerin ${ }^{\mathrm{a}, \mathrm{b}}$, Philippe Couvert ${ }^{\mathrm{a}, \mathrm{b}}$, Philippe Giral $^{\mathrm{a}, \mathrm{b}, \mathrm{c}, \mathrm{f}}$, Philippe Lesnik ${ }^{\mathrm{a}, \mathrm{b}}$, Wilfried Le Goff ${ }^{\mathrm{a}, \mathrm{b}}$, Isabelle Guillas ${ }^{\mathrm{a}, \mathrm{b}}$, Anatol Kontush ${ }^{\mathrm{a}, \mathrm{b}}$ *

${ }^{\text {a }}$ National Institute for Health and Medical Research (INSERM) UMR_S 1166, Faculty of Medicine Pitié-Salpétrière, 91 bld de L’Hopital, 75013 Paris, France; ' Sorbonne University, Paris, France; ${ }^{\text {c }}$ Institute of Cardiometabolism and Nutrition (ICAN), Paris, 75013 France; ${ }^{\mathrm{d}}$ Heart Institute-InCor, University of Sao Paulo, Sao Paulo, Brazil; ${ }^{\mathrm{e}}$ Laboratory of Lipids and Atherosclerosis, Department of Clinical Biochemistry, INFIBIOC, University of Buenos Aires, CONICET. Buenos Aires, Argentina; fAP-HP, Groupe hospitalier Pitié-Salpétrière, Paris, F-75013 France 


\section{Expanded Methods}

\section{Study populations}

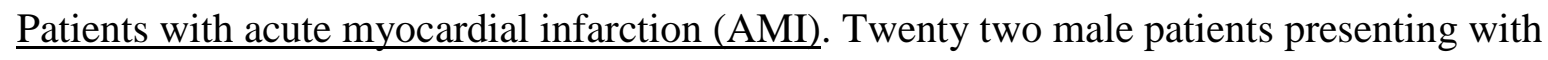
ST segment elevation myocardial infarction (STEMI) were recruited at the Heart InstituteInCor University of Sao Paulo Medical School Hospital (São Paulo, Brazil). All patients were recruited before initiation of any treatment within no later than $24 \mathrm{~h}$ of clinical presentation in the Emergency Room. The diagnosis of AMI was confirmed by clinical assessment by a cardiologist, including ECG changes, troponin I elevation, and the presence of coronary artery disease on coronary angiography in accordance with the Braunwald criteria. Exclusion criteria were female gender, secondary causes of hyperlipidemia (e.g. thyroid dysfunction), presence of inflammatory or infectious diseases, AMI or stroke during last six months, excessive alcohol consumption (>25 g/d), use of anti-inflammatory drugs (except aspirin) or antioxidant vitamins, and use of lipid-lowering drugs during the month preceding the clinical event. Comorbidities in the patient group included hypertension (77\%), Type 2 diabetes (18\%) and heart failure (18\%). Less than half of the patients were smokers (36\%). Eight healthy nonsmoking normolipidemic male subjects were recruited as controls. Only male subjects were included in the study to minimise hormonal effects on lipoprotein metabolism. Written informed consent was obtained from all study subjects and the project was approved by the Ethics Committee of InCor in accordance with local institutional guidelines conformed to the Declaration of Helsinki.

Patients with Type 2 diabetes (T2D). Seventeen treatment-naïve adult patients with T2D diagnosed according to the American Diabetes Association (ADA) criteria ${ }^{1}$ were recruited from the Ramon Carrillo Centre (La Matanza, Buenos Aires, Argentina). All diabetic patients were well-controlled and defined as those presenting HbA1c $<7 \%$ and fasting glucose $<7.22$ $\mathrm{mmol} / \mathrm{L}$ (130 mg/dL), following the ADA glycemic recommendations for patients with 
diabetes. ${ }^{1}$ Exclusion criteria were history of cardiovascular disease (CVD), concomitant kidney, thyroid or liver disease, excessive alcohol consumption (>25 g/d), smoking, and treatment with drugs affecting lipid or carbohydrates metabolism and/or with antioxidants during the last six months. Sixteen healthy non-smoking normolipidemic subjects who were either abstainers or moderate alcohol consumers ( $<25 \mathrm{~g} / \mathrm{d})$ were recruited from the same geographical area and with similar sociodemographic characteristics to constitute the control group. Only males and postmenopausal women not receiving hormone replacement therapy were included in the study to minimise hormonal effects on lipoprotein metabolism. Written informed consent was obtained from all patients and control subjects and the study was approved by the Ethics Committee of the Faculty of Pharmacy and Biochemistry, University of Buenos Aires, in accordance with guidelines conformed to the Declaration of Helsinki.

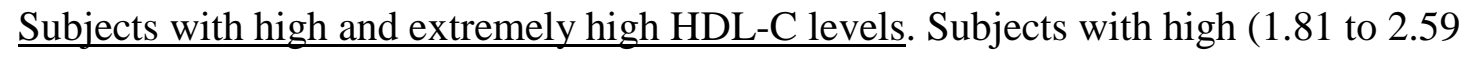
mmol/L [70 to $100 \mathrm{mg} / \mathrm{dL}] ; \mathrm{n}=20)$ and extremely high (>2.59 mmol/L [100 mg/dL]; n=20) HDL-C levels together with healthy normolipidemic control volunteers $(n=20)$ were recruited at the La Pitié-Salpétrière University Hospital (Paris, France). All control subjects were nonsmokers, and either abstainers or moderate alcohol consumers ( $<25 \mathrm{~g} / \mathrm{d})$. None of the control subjects presented renal, hepatic, gastrointestinal, pulmonary, endocrine, or oncological disease, or were receiving antioxidative vitamin supplementation or drugs known to affect lipoprotein metabolism for at least six weeks before the study. The study was performed in accordance with the ethical principles set forth in the Declaration of Helsinki and was approved by the Institutional Review Board of Hospital Pitié-Salpétrière. Written informed consent was obtained from all subjects.

\section{Blood samples}

Blood samples were withdrawn from the antecubital vein of each participant after an overnight fast. EDTA plasma (final EDTA concentration, $1 \mathrm{mg} / \mathrm{mL}$ ) was prepared from 
venous blood collected into sterile, evacuated tubes (Vacutainer). Plasma was immediately separated by low-speed centrifugation at $4{ }^{\circ} \mathrm{C}$, aliquoted and frozen at $-80{ }^{\circ} \mathrm{C}$ under nitrogen. To evaluate free cholesterol (FC) transfer to serum-derived HDL, serum samples were also obtained from some control subjects.

\section{Clinical and biological parameters}

Circulating levels of total cholesterol (TC), low-density lipoprotein-cholesterol (LDL-C), HDL-C, triglyceride (TG), glucose and HbA1c were measured using commercially available kits. Plasma apolipoprotein (apo) A-I and apoB were quantitated by immunoturbidimetry (DiaSys, France). High-sensitivity C-reactive protein (hsCRP) was measured by immunoassay (Roche Diagnostics, Mannheim, Germany). Activities of CETP and LCAT were measured in plasma using fluorometric kits from Roar Biomedical (New York, NY, USA).

\section{Isolation of lipoproteins}

Single-step ultracentrifugation (UC). To isolate TGRL ( $d<1.019 \mathrm{~g} / \mathrm{mL})$, a single-step UC was employed. ${ }^{2}$ Briefly, plasma was mixed with a required amount of dry solid $\mathrm{KBr}$ to increase its density to $1.019 \mathrm{~g} / \mathrm{mL}$, layered by a density solution of $1.019 \mathrm{~g} / \mathrm{mL}$ and ultracentrifuged at $45000 \mathrm{rpm}$ and $15^{\circ} \mathrm{C}$ for $24 \mathrm{~h}$. Upper layer containing TGRL was removed and extensively dialysed against phosphate-buffered saline (PBS; pH 7.4) at $4^{\circ} \mathrm{C}$ in the dark.

Density gradient UC. HDL was isolated from plasma by a single step, isopycnic non-denaturing density gradient UC using a Beckman SW41 Ti rotor at 40,000 rpm for 44 hours in a Beckman XL70 ultracentrifuge at $15^{\circ} \mathrm{C}$ by a slight modification of the method of Chapman et al. ${ }^{3}$ as previously described. ${ }^{4}$ Briefly, plasma density was increased to $1.21 \mathrm{~g} / \mathrm{mL}$ by adding dry solid $\mathrm{KBr}$. A discontinuous density gradient was then constructed as follows: 2 $\mathrm{mL}$ of $1.24 \mathrm{~g} / \mathrm{mL} \mathrm{NaCl} / \mathrm{KBr}$ solution, $3 \mathrm{~mL}$ plasma $(d 1.21 \mathrm{~g} / \mathrm{mL}), 2 \mathrm{~mL}$ of $1.063 \mathrm{~g} / \mathrm{mL}$ 
$\mathrm{NaCl} / \mathrm{KBr}$ solution, $2.5 \mathrm{~mL}$ of $1.019 \mathrm{~g} / \mathrm{mL} \mathrm{NaCl} / \mathrm{KBr}$ solution, and $2.5 \mathrm{~mL}$ of $\mathrm{NaCl}$ solution $(d 1.006 \mathrm{~g} / \mathrm{mL})$.

After centrifugation, gradients were collected from the top of the tube in fractions corresponding to VLDL and IDL ( $d<1.019 \mathrm{~g} / \mathrm{mL})$, LDL ( $d 1.019$ to $1.063 \mathrm{~g} / \mathrm{mL})$, HDL2b ( $d$ 1.063 to $1.091 \mathrm{~g} / \mathrm{mL}$ ), HDL2a (d 1.091 to $1.110 \mathrm{~g} / \mathrm{mL}$ ), HDL3a (d 1.110 to $1.133 \mathrm{~g} / \mathrm{mL}$ ), HDL3b ( $d 1.133$ to $1.156 \mathrm{~g} / \mathrm{mL}$ ), and HDL3c ( $d 1.156$ to $1.179 \mathrm{~g} / \mathrm{mL}$ ). Total HDL was prepared by reconstituting the five HDL subpopulations at their plasma concentrations. If necessary, the bottom of the tube containing plasma from control subjects was collected as lipoprotein-deficient plasma (LPDP). Density limits were defined using a calibration curve of density versus volume derived from control gradients containing only salt solutions. All fractions were extensively dialysed against $\mathrm{PBS}$ at $4^{\circ} \mathrm{C}$ in the dark before use.

ApoB depletion. To separate HDL from apoB-containing lipoproteins, apoB precipitating reagent containing phosphotungstic acid $(40 \mathrm{mg} / \mathrm{mL})$ and $\mathrm{MgCl}_{2}(100 \mathrm{mg} / \mathrm{mL})$ was added to plasma at a ratio of 1:10 v/v. The samples were incubated at room temperature for $10 \mathrm{~min}$ and centrifuged at $13000 \mathrm{rpm}$ for $30 \mathrm{~min}$. ApoB-depleted reference plasma was prepared by adding the apoB precipitant to a reference plasma sample obtained from a healthy normolipidemic male donor.

\section{Chemical composition of lipoproteins}

Total protein, TC, FC, phospholipid (PL) and TG contents of isolated lipoproteins were determined using commercially available assays. Cholesteryl ester (CE) was calculated by multiplying the difference between total and free cholesterol concentrations by 1.67. Total lipoprotein mass was calculated as the sum of total protein, CE, FC, PL and TG. Molecular masses of HDL2b and 3c of 450 and $160 \mathrm{kDa}$ and their protein contents of 28 and 58\%, respectively, were used to calculate molar concentrations of the particles. ${ }^{5,6}$ 
Measurement of HDL capacity to acquire FC during TGRL lipolysis by LPL

Fluorescent 23-(dipyrrometheneboron difluoride)-24-norcholesterol (Avanti Polar Lipids, Alabaster, AL, USA), also known as TopFluor® cholesterol (TopF) and BODIPYcholesterol, was employed to trace the lipolytic transfer of FC to HDL during TGRL lipolysis. Human TGRLs were labelled with TopF and incubated in the presence of LPL and HDL at $37^{\circ} \mathrm{C}$. At the end of the incubation, the lipoproteins were separated and HDL was analysed for the presence of TopF.

To label human TGRL with TopF, TGRL was added to lipoprotein-deficient plasma (LPDP) at the LPDP:TGRL ratio of 1:100 by volume. The mixture was filtered with $0.8 \mu \mathrm{m}$ filter (RC Membrane, Phenomenex, USA), the tube was covered with aluminium foil and TopF was added as a chloroformic solution followed by overnight incubation at $37^{\circ} \mathrm{C}$ under gentle stirring. Labelled TGRL was separated from unbound fluorescent lipid using filtration through a PD-10 Sephadex column (GE Healthcare, SephadexTM G-25M, UK). Absence in the eluate of other, than TGRL, material containing fluorescent lipid was confirmed by FPLC. Triglyceride concentration in purified labelled TGRL was measured by photometry and TopF fluorescence was registered at the excitation/emission wavelengths of 500/525 nm using Gemini Microplate Reader (Molecular Devices, USA) to verify the labelling. Briefly, a specific TopF fluorescence per unit TG concentration was calculated and TGRL samples revealing the specific fluorescence below a pre-defined threshold were discarded.

To evaluate the transfer of TopF, Tris buffer $(0.4 \mathrm{M}$, $\mathrm{pH}$ 8) was mixed on ice with required amounts of TopF-labelled TGRL and HDL, and LPL from Pseudomonas sp. or from bovine milk (both from Sigma, France) was added to start lipolysis at $37^{\circ} \mathrm{C}$. At the end of the incubation, the reaction mixture was placed on ice and apoB precipitant containing phosphotungstic acid and $\mathrm{MgCl}_{2}$ was added. The time-point corresponding to $0 \mathrm{~h}$ was prepared by placing the mixture on ice immediately after adding LPL. Following incubation at room 
temperature and centrifugation, HDL-containing supernatant was aspirated, filtered through a $0.45 \mu \mathrm{m}$ filter (RC Membrane, Phenomenex, USA) and transferred to a black microplate for fluorescence reading as described above. Fluorescence of the TGRL sample alone was also measured at a triglyceride concentration employed in the assay and fluorescent values measured in HDL were expressed as a percentage of fluorescence of such standard sample. To evaluate effects on the transfer of specific inhibitors for CETP and LCAT, the assay was performed in the absence or presence of torcetrapib (25 $\mu \mathrm{M})$ or iodoacetamide (750 $\mu \mathrm{M})$.

To characterise the transfer of TopF to HDL obtained from clinical plasma samples, TGRL and apoB-depleted plasma were used at the final concentration of $0.34 \mathrm{mmol} / \mathrm{L}$ (30 mg triglyceride/dL) and dilution of 30-fold, respectively. Lipid concentrations were evaluated in the samples using commercially available kits. Assay conditions (lipoprotein concentrations, incubation time, etc.) were chosen to model those of postprandial TGRL lipolysis in humans. A reference apoB-depleted plasma sample obtained from one healthy normolipidemic control plasma aliquoted and stored at $-80^{\circ} \mathrm{C}$ was included in each series of measurements and all values obtained in clinical samples were normalised to that observed in the reference plasma. In some experiments, transfer of FC was evaluated fluorometrically using Amplex ${ }^{\circledR}$ Red kit (ThermoFisher Scientific, Montigny-le-Bretonneux, France).

\section{Postprandial aortic cholesterol accumulation in mice}

Postprandial aortic accumulation of cholesterol was evaluated in vivo after a gavage with 100 $\mu \mathrm{Ci}(3.7 \mathrm{MBq})$ of $\left[{ }^{3} \mathrm{H}\right]$-cholesterol administrated with olive oil $(100 \mu \mathrm{L})$ to three groups of mice greatly differing in HDL-C levels, including low-HDL-C apoA-I knock-out mice (SN 002055 B6.129P2-Apoa1tm1Unc/J), high-HDL-C human apoA-I transgenic mice (SN 001927 C57BL/6-Tg(APOA1)1Rub/J) and control wild-type mice (C57BL/6, all strains from Charles River Laboratories, L’Arbresle, France). The groups contained similar numbers of males and females and the sex ratio did not differ between the groups. The animals were 
euthanized $2 \mathrm{~h}$ after the gavage, their aortas removed and weighted, and specific radioactivity measured using a MicroBeta 2450 Perkin-Elmer liquid scintillation counter.

The capacity of murine HDL to acquire TopF from TGRL during LPL-induced lipolysis was evaluated in apoB-depleted EDTA plasma obtained from the animals several weeks before the gavage experiment, following an overnight fast. All animal experiments were approved by the local ethics committee for animal care.

\section{Statistical analysis}

Incubations were performed in duplicates. As several individual samples were employed in every experiment, experimental errors reported primarily reflect biological variability across individuals rather than experimental reproducibility.

Distributions of all variables were analysed for normality using Kolmogorov-Smirnov test. Normally distributed variables are expressed as means \pm SD and non-Gaussian distributed variables are expressed as median (interquartile range). Between-groups differences in normally distributed variables were analysed using Student's t-test. For non-Gaussian distributed variables, the non-parametric Mann-Whitney U test was employed. Differences in dichotomous variables were analyzed by Fisher's exact test.

Spearman's correlation coefficients were calculated to evaluate relationships between variables. The statistical software employed was Statistica (StatSoft, Tulsa, OK, USA).

The ggplot2 package from $\mathrm{R}$ was employed to graphically represent relationships between means \pm SDs of two variables across several groups of subjects. 


\section{References}

1. Executive summary: Standards of medical care in diabetes--2013. Diabetes Care 2013;36 Suppl 1:S4-10.

2. Guerin M, Dolphin PJ and Chapman MJ. Preferential cholesteryl ester acceptors among the LDL subspecies of subjects with familial hypercholesterolemia. Arterioscler Thromb 1994;14:679-685.

3. Chapman MJ, Goldstein S, Lagrange D and Laplaud PM. A density gradient ultracentrifugal procedure for the isolation of the major lipoprotein classes from human serum. J Lipid Res 1981;22:339-58.

4. Guerin M, Bruckert E, Dolphin PJ, Turpin G and Chapman MJ. Fenofibrate reduces plasma cholesteryl ester transfer from HDL to VLDL and normalizes the atherogenic, dense LDL profile in combined hyperlipidemia. Arterioscler Thromb Vasc Biol 1996;16:763-72. 5. Rached F, Lhomme M, Camont L, Gomes F, Dauteuille C, Robillard P, Santos RD, Lesnik P, Serrano CV, Jr., Chapman MJ and Kontush A. Defective functionality of small, dense HDL3 subpopulations in ST segment elevation myocardial infarction: Relevance of enrichment in lysophosphatidylcholine, phosphatidic acid and serum amyloid A. Biochim Biophys Acta 2015;1851:1254-61.

6. Kontush A and Chapman MJ. High-Density Lipoproteins: Structure, Metabolism, Function and Therapeutics. New York: Wiley \& Sons; 2012. 
Supplemental Table 1. Clinical and biological characteristics of patients with AMI and healthy controls

\begin{tabular}{|c|c|c|}
\hline & Controls (n=8) & AMI $(n=22)$ \\
\hline Age (years) & $53 \pm 11$ & $57 \pm 10$ \\
\hline $\operatorname{Sex}(M / F)$ & $8 / 0$ & $22 / 0$ \\
\hline BMI $\left(\mathrm{kg} / \mathrm{m}^{2}\right)$ & $23.6 \pm 3.0$ & $26.6 \pm 4.0$ \\
\hline Glucose (mg/dL) & $97 \pm 11$ & $134 \pm 67$ \\
\hline$(\mathrm{mmol} / \mathrm{L})$ & $5.38 \pm 0.61$ & $7.44 \pm 3.72$ \\
\hline TC (mg/dL) & $188 \pm 21$ & $202 \pm 57$ \\
\hline$(\mathrm{mmol} / \mathrm{L})$ & $4.86 \pm 0.54$ & $5.22 \pm 1.47$ \\
\hline TG (mg/dL) & $68 \pm 16$ & $146 \pm 98 *$ \\
\hline$(\mathrm{mmol} / \mathrm{L})$ & $0.77 \pm 0.18$ & $1.64 \pm 1.11^{*}$ \\
\hline LDL-C (mg/dL) & $118 \pm 26$ & $131 \pm 45$ \\
\hline$(\mathrm{mmol} / \mathrm{L})$ & $3.05 \pm 0.67$ & $3.39 \pm 1.16$ \\
\hline VLDL-C (mg/dL) & $16 \pm 7$ & $28 \pm 19$ \\
\hline$(\mathrm{mmol} / \mathrm{L})$ & $0.41 \pm 0.18$ & $0.72 \pm 0.49$ \\
\hline ApoB-100 (mg/dL) & $90 \pm 24$ & $98 \pm 36$ \\
\hline$(\mathrm{mmol} / \mathrm{L})$ & $0.0018 \pm 0.0005$ & $0.0019 \pm 0.0007$ \\
\hline HDL-C (mg/dL) & $54 \pm 8$ & $39 \pm 7 * * *$ \\
\hline$(\mathrm{mmol} / \mathrm{L})$ & $1.40 \pm 0.21$ & $1.01 \pm 0.18 * * *$ \\
\hline ApoA-I (mg/dL) & $143 \pm 13$ & $114 \pm 20 * *$ \\
\hline$(\mathrm{mmol} / \mathrm{L})$ & $0.05 \pm 0.005$ & $0.04 \pm 0.007^{* *}$ \\
\hline Non HDL cholesterol (mg/dL) & $122 \pm 41$ & $163 \pm 58$ \\
\hline$(\mathrm{mmol} / \mathrm{L})$ & $3.16 \pm 1.06$ & $4.22 \pm 1.50$ \\
\hline hsCRP (mg/L) & $0.53(0.16 ; 5.25)$ & $6.03(0.62 ; 47.68) * * *$ \\
\hline$(\mathrm{nmol} / \mathrm{L})$ & $5.10(1.52 ; 50)$ & $57.4(5.90 ; 454.10) * * *$ \\
\hline
\end{tabular}

AMI, acute myocardial infraction; apoA-I, apolipoprotein A-I; apoB, apolipoprotein B; BMI, body mass index; HDL, high-density lipoprotein; HDLC, high-density lipoprotein-cholesterol; hsCRP, highly sensitive C-reactive protein; LDL-C, low-density lipoprotein-cholesterol; TC, total cholesterol; TG, triglycerides; VLDL-C, very low-density lipoprotein-cholesterol; *** $\mathrm{p}<0.001$, ${ }^{* *} \mathrm{p}<0.01,{ }^{*} \mathrm{p}<0.05$ vs. controls. 
Supplemental Table 2. Clinical and biological characteristics of patients with T2D and healthy controls

\begin{tabular}{ccc}
\hline & Controls $(\mathrm{n}=16)$ & T2D $(\mathrm{n}=17)$ \\
\hline Age (years) & $53 \pm 10$ & $56 \pm 10$ \\
Sex (M/F) & $8 / 8$ & $6 / 11$ \\
Waist circumference (cm) & $94 \pm 14$ & $101 \pm 14$ \\
BMI (kg/m²) & $26.5 \pm 4.4$ & $30.5 \pm 4.7^{*}$ \\
Glucose (mg/dL) & $80 \pm 9$ & $129 \pm 32^{* * *}$ \\
(mmol/L) & $4.44 \pm 0.50$ & $7.16 \pm 1.78^{* * *}$ \\
TC (mg/dL) & $204 \pm 34$ & $197 \pm 40^{*}$ \\
$(\mathrm{mmol} / \mathrm{L})$ & $5.28 \pm 0.88$ & $5.09 \pm 1.03^{*}$ \\
$\mathrm{TG}(\mathrm{mg} / \mathrm{dL})$ & $93 \pm 42$ & $180 \pm 133^{*}$ \\
$(\mathrm{mmol} / \mathrm{L})$ & $1.05 \pm 0.47$ & $2.03 \pm 1.50^{*}$ \\
LDL-C (mg/dL) & $131 \pm 34$ & $124 \pm 41$ \\
$(\mathrm{mmol} / \mathrm{L})$ & $3.39 \pm 0.88$ & $3.21 \pm 1.06$ \\
ApoB-100 (mg/dL) & $99 \pm 45$ & $130 \pm 76$ \\
$(\mathrm{mmol} / \mathrm{L})$ & $0.0019 \pm 0.0009$ & $0.0025 \pm 0.0015$ \\
HDL-C (mg/dL) & $53 \pm 13$ & $42 \pm 11^{*}$ \\
$(\mathrm{mmol} / \mathrm{L})$ & $1.37 \pm 0.34$ & $1.09 \pm 0.28^{*}$ \\
ApoA-I (mg/dL) & $169 \pm 60$ & $142 \pm 31$ \\
$(\mathrm{mmol} / \mathrm{L})$ & $0.06 \pm 0.02$ & $0.05 \pm 0.01$ \\
$\mathrm{HbA} 1 \mathrm{c}$ & $4.99 \pm 0.72$ & $6.09 \pm 0.61^{* * *}$ \\
$\mathrm{hsCRP}(\mathrm{mg} / \mathrm{L})$ & $0.38(0.17 ; 2.63)$ & $1.135(0.46 ; 5.08)$ \\
$(\mathrm{nmol} / \mathrm{L})$ & $3.62(1.62 ; 25.05)$ & $10.81(4.38 ; 48.38)$ \\
\hline & &
\end{tabular}

apoA-I, apolipoprotein A-I; apoB, apolipoprotein B; BMI, body mass index; HDL, high-density lipoprotein; HDL-C, high-density lipoprotein-cholesterol; hsCRP, highly sensitive C-reactive protein; LDL-C, low-density lipoproteincholesterol; T2D, Type 2 diabetes; TC, total cholesterol; TG, triglycerides; *** $\mathrm{p}<0.001,{ }^{*} \mathrm{p}<0.05$ vs. controls. 
Supplemental Table 3. Clinical and biological characteristics of subjects with high HDL-C (1.81 to $2.59 \mathrm{mmol} / \mathrm{L}$ [70 to $100 \mathrm{mg} / \mathrm{dL}$ ]) and healthy normolipidemic controls

\begin{tabular}{rcc}
\hline & Controls $(\mathrm{n}=10)$ & High HDL-C $(\mathrm{n}=20)$ \\
\hline Age (years) & $47 \pm 14$ & $56 \pm 13$ \\
Sex (M/F) & $3 / 7$ & $4 / 16$ \\
TC (mg/dL) & $197 \pm 28$ & $236 \pm 47^{*}$ \\
$(\mathrm{mmol} / \mathrm{L})$ & $5.09 \pm 0.72$ & $6.10 \pm 1.22^{*}$ \\
TG $(\mathrm{mg} / \mathrm{dL})$ & $62 \pm 17$ & $84 \pm 34$ \\
$(\mathrm{mmol} / \mathrm{L})$ & $0.70 \pm 0.20$ & $0.95 \pm 0.38$ \\
LDL-C (mg/dL) & $121 \pm 33$ & $139 \pm 45$ \\
$(\mathrm{mmol} / \mathrm{L})$ & $3.13 \pm 0.85$ & $3.60 \pm 1.16$ \\
HDL-C (mg/dL) & $61 \pm 15$ & $80 \pm 7 * * *$ \\
$(\mathrm{mmol} / \mathrm{L})$ & $1.58 \pm 0.39$ & $2.07 \pm 0.18^{* * *}$ \\
\hline
\end{tabular}

HDL-C, high-density lipoprotein-cholesterol; LDL-C, low-density lipoprotein-cholesterol; TC, total cholesterol; TG, triglycerides; *** $\mathrm{p}<0.001,{ }^{*} \mathrm{p}<0.05$ vs. controls. 
Supplemental Table 4. Clinical and biological characteristics of subjects with extremely high HDL-C (>2.59 mmol/L [>100 mg/dL]) and healthy normolipidemic controls

\begin{tabular}{rcc}
\hline & Controls $(\mathrm{n}=10)$ & Extremely high HDL-C $(\mathrm{n}=20)$ \\
\hline Age (years) & $44 \pm 13$ & $66 \pm 9 * *$ \\
Sex $(\mathrm{M} / \mathrm{F})$ & $4 / 6$ & $3 / 17$ \\
$\mathrm{TC}(\mathrm{mg} / \mathrm{dL})$ & $195 \pm 30$ & $268 \pm 55^{*}$ \\
$(\mathrm{mmol} / \mathrm{L})$ & $5.05 \pm 0.78$ & $6.94 \pm 1.42^{*}$ \\
$\mathrm{TG}(\mathrm{mg} / \mathrm{dL})$ & $73 \pm 13$ & $74 \pm 32$ \\
$(\mathrm{mmol} / \mathrm{L})$ & $0.82 \pm 0.15$ & $0.84 \pm 0.36$ \\
$\mathrm{LDL}-\mathrm{C}(\mathrm{mg} / \mathrm{dL})$ & $118 \pm 35$ & $136 \pm 53$ \\
$(\mathrm{mmol} / \mathrm{L})$ & $3.06 \pm 0.91$ & $3.52 \pm 1.37$ \\
HDL-C (mg/dL) & $61 \pm 15$ & $117 \pm 19^{* * *}$ \\
$(\mathrm{mmol} / \mathrm{L})$ & $1.58 \pm 0.39$ & $3.03 \pm 0.49 * * *$ \\
\hline
\end{tabular}

HDL-C, high-density lipoprotein-cholesterol; LDL-C, low-density lipoproteincholesterol; TC, total cholesterol; TG, triglycerides; ${ }^{* * *} \mathrm{p}<0.001,{ }^{* *} \mathrm{p}<0.01$, * $\mathrm{p}<0.05$ vs. controls. 


\section{Supplemental Figure Legends}

\section{Supplemental Figure 1. Transfer of fluorescent FC from TGRL to HDL during LPL-}

induced lipolysis. HDL was isolated from normolipidemic EDTA plasma by density gradient ultracentrifugation (A) or by apoB depletion (B) and incubated for up to $3 \mathrm{~h}$ at $37^{\circ} \mathrm{C}$ with LPL (190 U/mL) and TGRL (30 mg triglyceride/dL) labelled with TopF at a final concentration of 2.5 to $20 \mathrm{mg}$ HDL protein/dL or a final concentration of apoB-depleted plasma of 2.5 to $20 \%$ v/v, respectively. At the end of the incubation, HDL was separated from TGRL by apoB depletion and TopF fluorescence was measured in HDL at the excitation/emission wavelengths of 500/525 nm. Data from four to six independent experiments performed in duplicate with four individual HDL samples are shown; ${ }^{* * *} \mathrm{p}<0.001,{ }^{* *} \mathrm{p}<0.01$, ${ }^{*} \mathrm{p}<0.05$ vs. $0 \mathrm{mg} / \mathrm{dL} ; \dagger \dagger$ p $<0.01, \dagger p<0.05$ vs. $2.5 \mathrm{mg} / \mathrm{dL} ; \ddagger \mathrm{p}<0.05$ vs. $5 \mathrm{mg} / \mathrm{dL}$ (A); ${ }^{* * *}$ p $<0.001$, ${ }^{* *} \mathrm{p}<0.01,{ }^{*} \mathrm{p}<0.05$ vs. $0 \%$; $\dagger \mathrm{p}<0.05$ vs. $2.5 \%$; $\ddagger \mathrm{p}<0.05$ vs. $5 \%$ (B).

\section{Supplemental Figure 2. Dose-dependences of the accumulation of fluorescent TopF} cholesterol and unlabelled FC in HDL after LPL-induced TGRL lipolysis. HDL was isolated from normolipidemic EDTA plasma by apoB depletion and incubated for $2 \mathrm{~h}$ at $37^{\circ} \mathrm{C}$ with LPL (190 U/mL) and either TGRL labelled with TopF (A) or non-labelled TGRL (B; both at $15 \mathrm{mg}$ TG/dL), at final dilutions of apoB-depleted plasma of 2.5, 5, 10 and 20\%. At the end of the incubation, HDL was separated from TGRL by apoB depletion, and TopF fluorescence or FC were measured in HDL at 500/525 nm or by the Amplex Red kit, respectively. Data from three to four independent experiments performed in duplicate with three individual HDL samples are shown; ** $<<0.01,{ }^{*} \mathrm{p}<0.05$ vs. $0 \%{ }^{{ }^{\dagger \dagger}} \mathrm{p}<0.01,{ }^{\dagger} \mathrm{p}<0.05$ vs. $2.5 \% ;{ }^{\ddagger} \mathrm{p}<0.01,{ }^{\ddagger} \mathrm{p}<0.05$ vs. $5 \%$.

\section{Supplemental Figure 3. Transfer of FC from TGRL during LPL-induced lipolysis to}

HDL isolated from patients with AMI. HDL was isolated by UC from patients with AMI $(\mathrm{n}=7)$ and healthy normolipidemic controls $(\mathrm{n}=6)$ and TopF transfer to HDL (4 mg 
protein/dL) from TGRL (30 mg TG/dL) was evaluated (A). In a separate experiment, HDL was isolated by apoB depletion from patients with AMI ( $\mathrm{n}=7)$ and healthy normolipidemic controls ( $\mathrm{n}=7$ ) and FC transfer from TGRL (30 mg TG/dL) was evaluated by the Amplex Red kit at the concentration of apoB-depleted plasma of 3.3\% (B); ** p $<0.01$ vs. HDL from controls.

\section{Supplemental Figure 4. Activities of CETP (A) and LCAT (B) in plasma of patients with} AMI, T2D, extremely high HDL-C and healthy normolipidemic controls. The measurements were performed using kits from Roar Biomedical in ten patients randomly selected from each group; * $\mathrm{p}<0.05$ vs. controls; a. u., arbitrary units.

Supplemental Figure 5. Transfer of fluorescent FC to HDL subpopulations during LPLinduced lipolysis of TGRL. Five major HDL subpopulations were isolated from normolipidemic EDTA plasma by density gradient UC and incubated at a final concentration of $4 \mathrm{mg}$ HDL protein/dL (equivalent to 0.32 and $0.43 \mu \mathrm{M}$ of HDL2b and 3c, respectively) for $2 \mathrm{~h}$ at $37^{\circ} \mathrm{C}$ with LPL (190 U/mL) and TopF-labelled TGRL (30 mg TG/dL). At the end of the incubation, HDL was separated from TGRL by apoB depletion and TopF fluorescence was measured in HDL at the excitation/emission wavelengths of 500/525 nm. Data from four independent experiments performed with four individual HDL samples are shown; **p < 0.01, ${ }^{*} \mathrm{p}<0.05$ vs. HDL2b; ${ }^{\dagger} \mathrm{p}<0.01,{ }^{\dagger} \mathrm{p}<0.05$ vs. HDL2a; ${ }^{\ddagger \ddagger} \mathrm{p}<0.01,{ }^{\ddagger} \mathrm{p}<0.05$ vs. HDL3a; ${ }^{\S \S} \mathrm{p}<0.01$ vs. HDL3b.

\section{Supplemental Figure 6. Relationships of overall and cardiovascular mortality with} plasma HDL-C levels and HDL capacity to acquire TopF from TGRL upon LPLinduced lipolysis. Sex-adjusted and age-standardized cardiovascular mortality (per 1000 person-years) was calculated using data from the CANHEART Study (1) and Copenhagen City Heart Study (CCHS) (2) for mean HDL-C levels observed in the five populations studied (AMI, T2D, high HDL-C, extremely high HDL-C, and all controls combined) and plotted 
against the means \pm SDs of HDL-C levels in these populations using ggplot2 package from $\mathrm{R}$ (A), or against means \pm SDs of HDL capacity to acquire TopF using orthogonal regression (B). Note that orthogonal fitting was impossible to perform in the panel A, reflecting p-values of $>0.05$ for the correlations between mortality and HDL-C. Data points are colour-coded according to the axis titles.

\section{Supplemental Figure 7. Activities of CETP (A) and LCAT (B) in plasma of human} apoA-I transgenic, apoA-I knock-out and wild-type mice. Values obtained in healthy normolipidemic human controls are shown for comparison. The measurements were performed using kits from Roar Biomedical in three mice randomly selected from each group; ${ }^{* * *} \mathrm{p}<0.001,{ }^{* *} \mathrm{p}<0.01,{ }^{*} \mathrm{p}<0.05$ vs. human controls; ${ }^{\dagger} \mathrm{p}<0.05$ vs. wild-type mice; a. u., arbitrary units.

\section{References}

1. Ko DT, Alter DA, Guo H, Koh M, Lau G, Austin PC, Booth GL, Hogg W, Jackevicius CA, Lee DS, et al. High-Density Lipoprotein Cholesterol and CauseSpecific Mortality in Individuals Without Previous Cardiovascular Conditions: The CANHEART Study. J Am Coll Cardiol. 2016;68(19):2073-83.

2. Madsen CM, Varbo A, and Nordestgaard BG. Extreme high high-density lipoprotein cholesterol is paradoxically associated with high mortality in men and women: two prospective cohort studies. Eur Heart J. 2017;38(32):2478-86. 

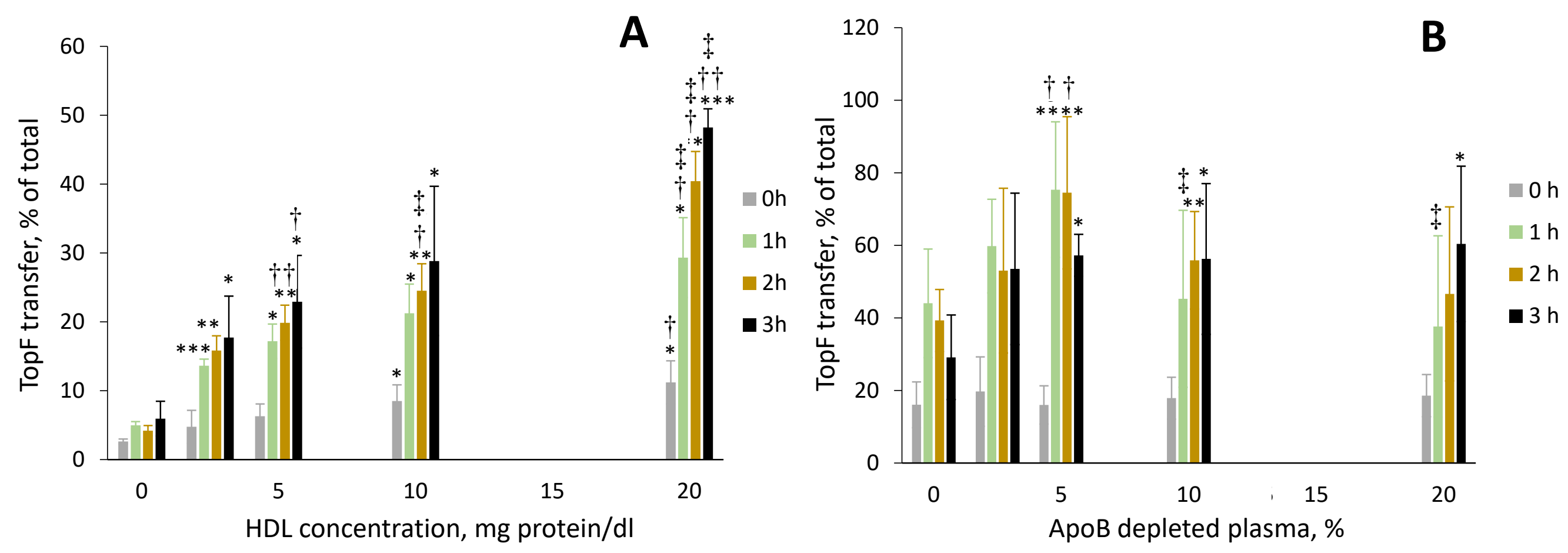
A

B
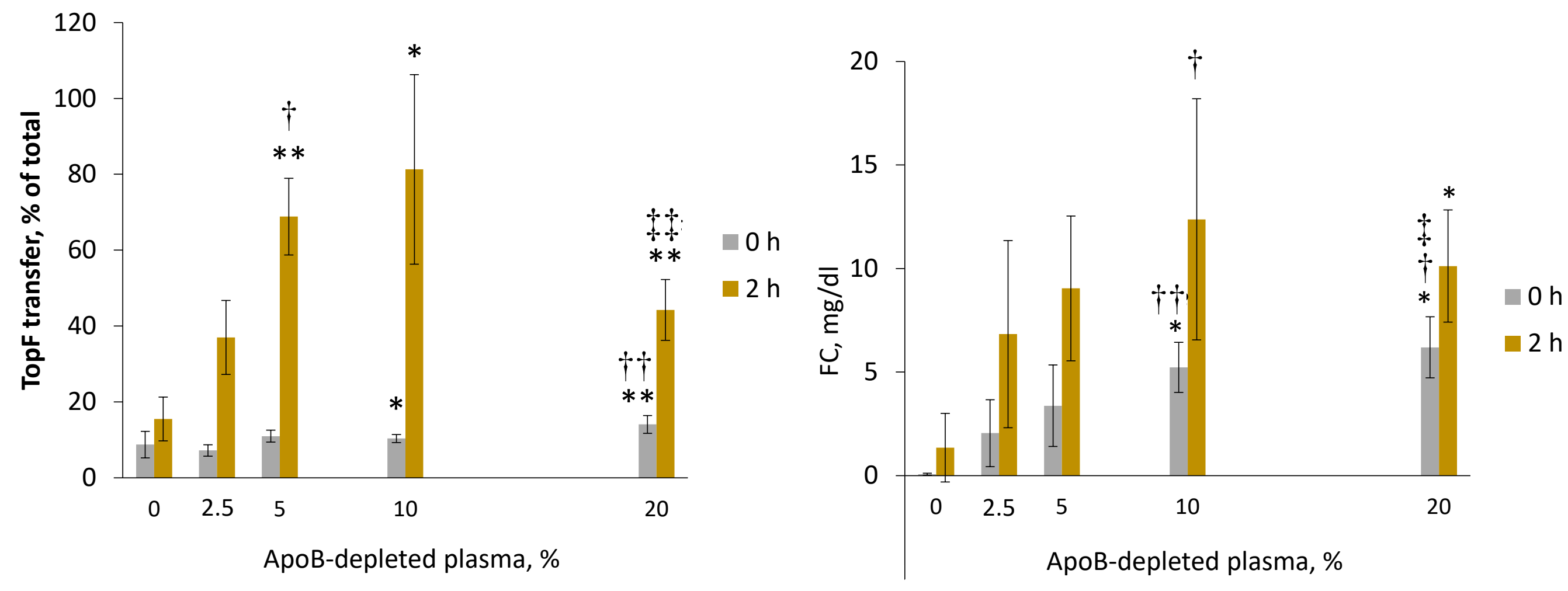

Supplemental Figure 2 


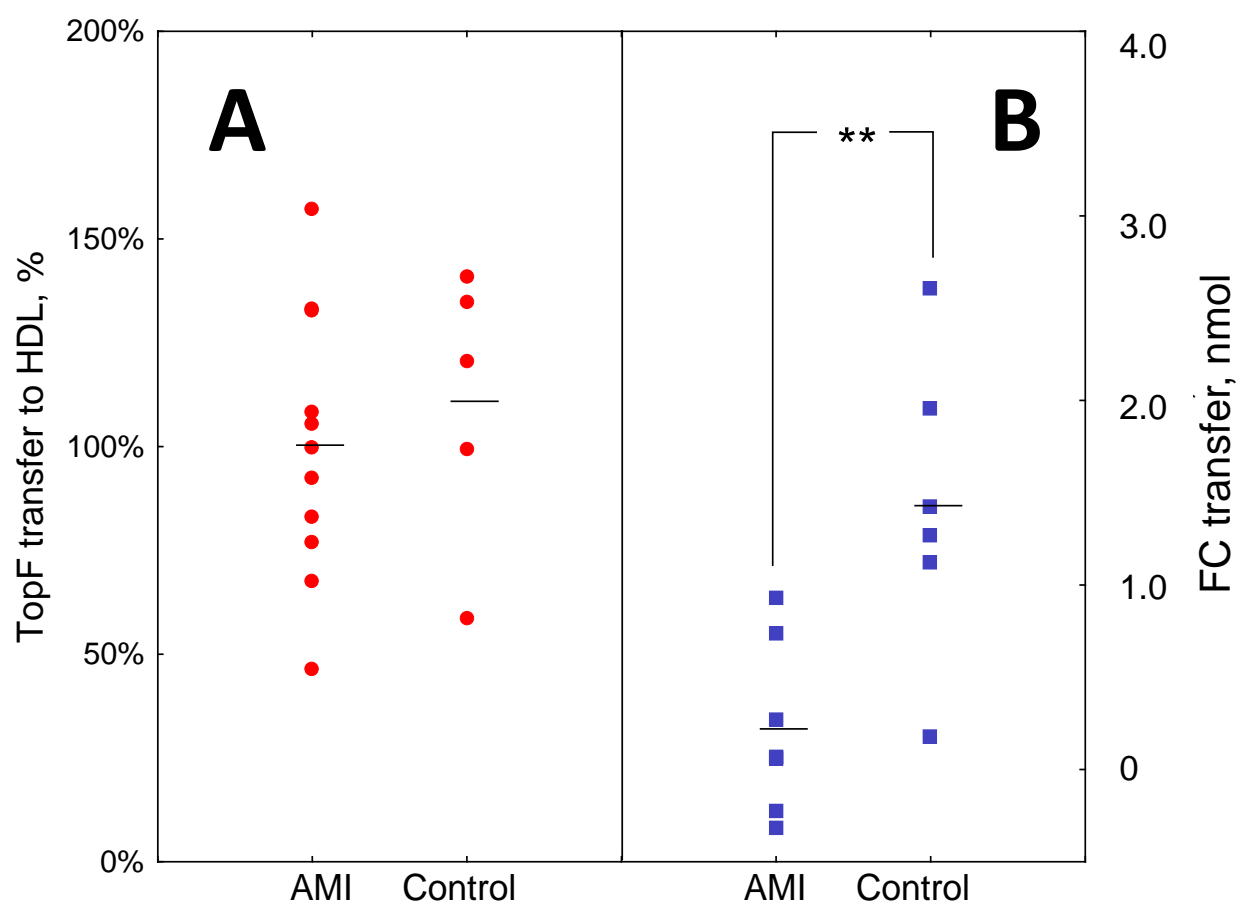

Supplemental Figure 3 

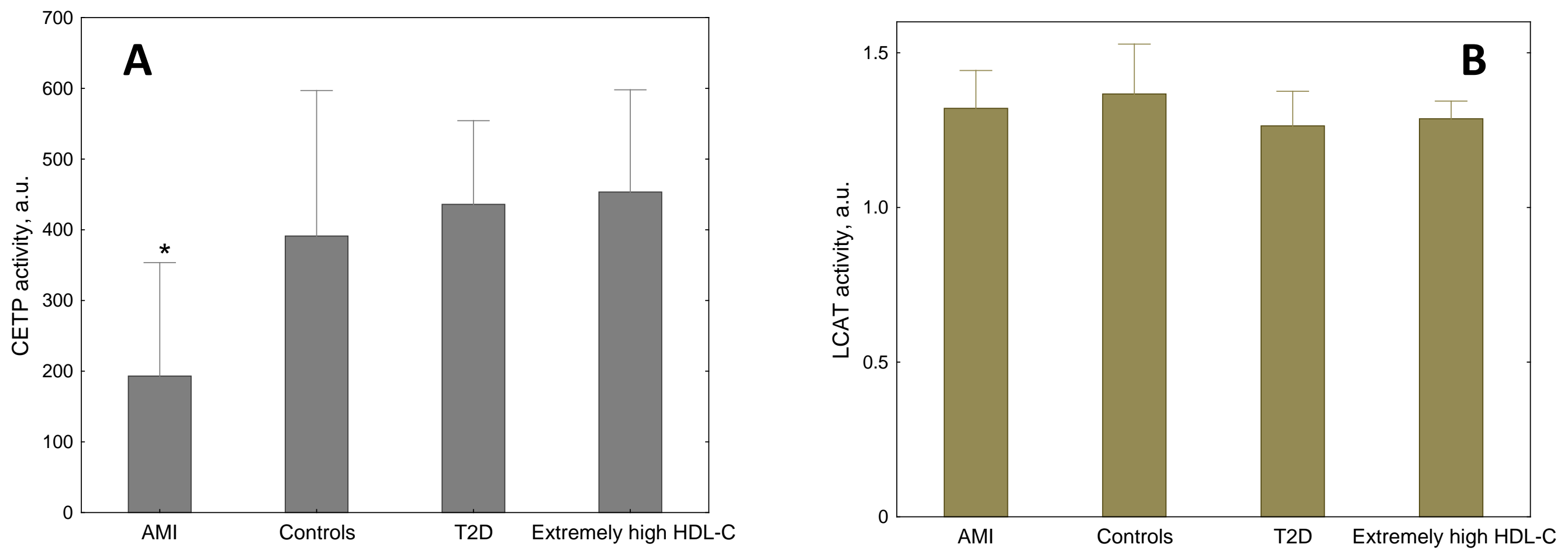


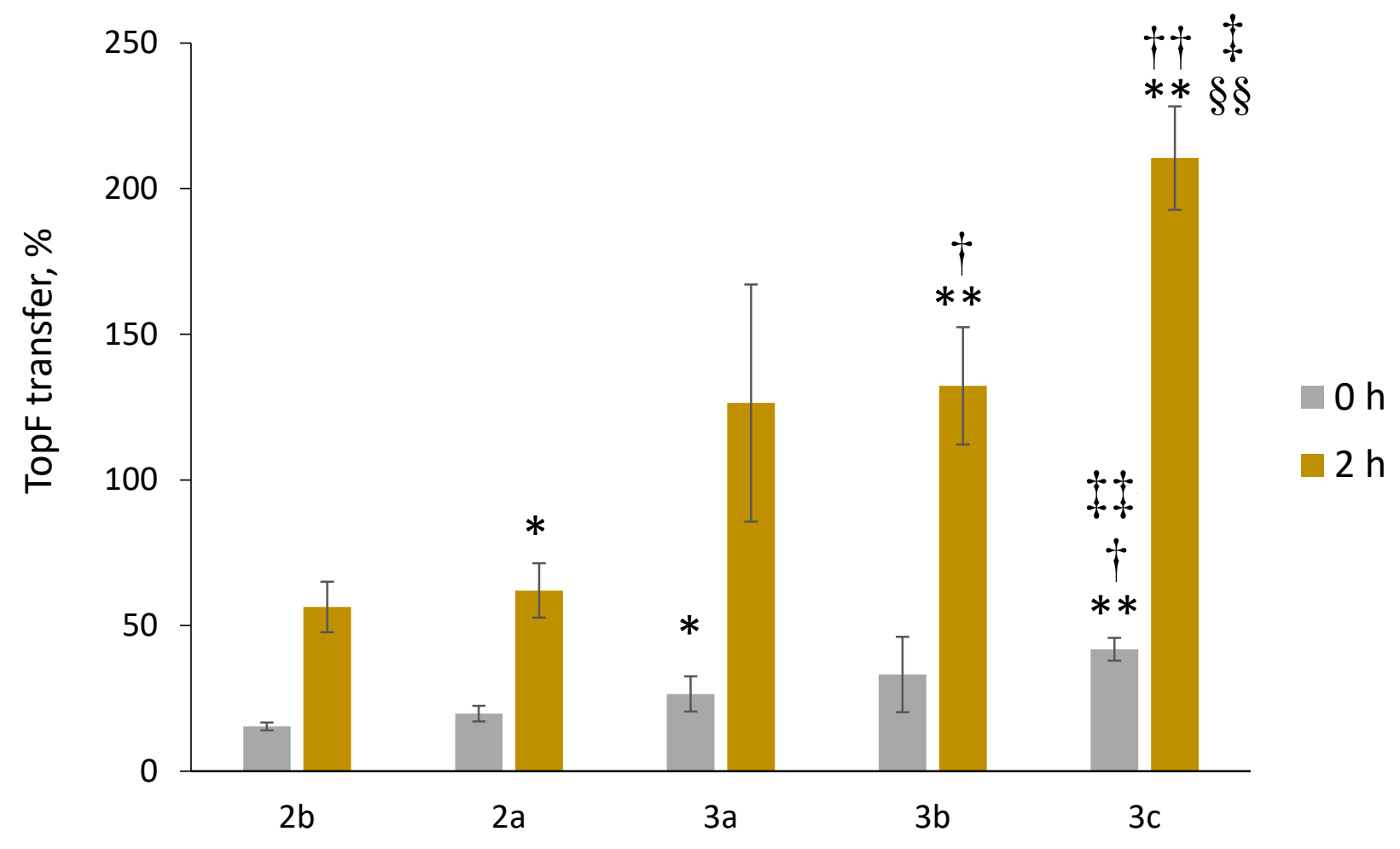

Supplemental Figure 5 

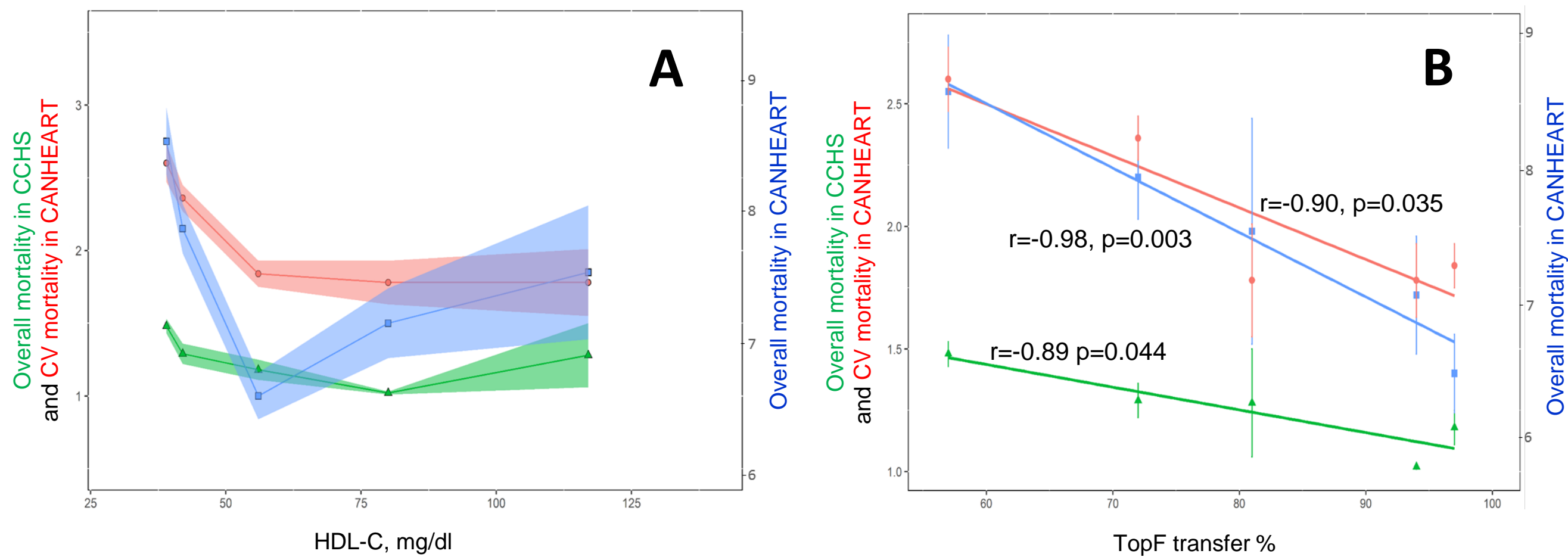
